\title{
Direct numerical simulation and large-eddy simulation of stationary buoyancy-driven turbulence
}

\author{
D. CHUNG† AND D. I. PULLIN \\ Graduate Aerospace Laboratories, California Institute of Technology, Pasadena, CA 91125, USA
}

(Received 10 June 2009; revised 15 October 2009; accepted 16 October 2009; first published online 24 December 2009)

We report direct numerical simulation (DNS) and large-eddy simulation (LES) of statistically stationary buoyancy-driven turbulent mixing of an active scalar. We use an adaptation of the fringe-region technique, which continually supplies the flow with unmixed fluids at two opposite faces of a triply periodic domain in the presence of gravity, effectively maintaining an unstably stratified, but statistically stationary flow. We also develop a new method to solve the governing equations, based on the Helmholtz-Hodge decomposition, that guarantees discrete mass conservation regardless of iteration errors. Whilst some statistics were found to be sensitive to the computational box size, we show, from inner-scaled planar spectra, that the small scales exhibit similarity independent of Reynolds number, density ratio and aspect ratio. We also perform LES of the present flow using the stretched-vortex subgridscale (SGS) model. The utility of an SGS scalar flux closure for passive scalars is demonstrated in the present active-scalar, stably stratified flow setting. The multi-scale character of the stretched-vortex SGS model is shown to enable extension of some second-order statistics to subgrid scales. Comparisons with DNS velocity spectra and velocity-density cospectra show that both the resolved-scale and SGS-extended components of the LES spectra accurately capture important features of the DNS spectra, including small-scale anisotropy and the shape of the viscous roll-off.

Key words: modelling, simulation, turbulent mixing

\section{Introduction}

The buoyancy-driven turbulent mixing of variable-density fluids arises in many applications, ranging from the naturally occurring exploding supernovae to the manmade inertial confinement fusion, and from the weighty subject of environmental pollution to the whimsical emptying of an inverted glass of water (Sandoval 1995; Cook \& Dimotakis 2001; Dimotakis 2005). To better understand and predict these flows, researchers have proposed various ways to capture the essential physics of these flows in simple models that enable academic investigation through laboratory experiments, numerical simulations and theoretical development.

In the spirit of such efforts, we propose a new model for the simulation of statistically stationary buoyancy-driven turbulent mixing of a variable-density fluid

$\dagger$ Email address for correspondence: dchung@caltech.edu 
by employing a fringe region (Bertolotti, Herbert \& Spalart 1992; Nordström, Nordin \& Henningson 1999), which sustains an unstable density gradient within a triply periodic domain. Following Sandoval (1995), we consider an incompressible binary fluid mixture composed of fluids with microscopic densities $\rho_{1}$ and $\rho_{2}$, with the convention $\rho_{2}>\rho_{1}$. Presently, we are interested in moderately high density ratios $R$ ( $\equiv \rho_{2} / \rho_{1}$ ), namely $R=3$ and 7, a regime in which the Boussinesq assumption, formally $R=1$, is no longer valid. The present model draws on many loosely related ideas from the literature; we will highlight some important similarities and differences in the following.

Overholt \& Pope (1996) and Yeung, Donzis \& Sreenivasan (2005) simulated, in a triply periodic domain, the mixing of a passive scalar by forced isotropichomogeneous turbulence embedded in background mean scalar gradient. Passive scalar fluctuations are continually produced by the scalar flux working against the background scalar gradient, but are kept in balance by diffusive dissipation, resulting in a statistically stationary flow. While, like Overholt \& Pope (1996), our present model can also be viewed as a statistically stationary scalar-mixing flow in a background scalar gradient, there are two important distinctions. First, the present model considers an active scalar, the mass fraction (algebraically related to the density), whose spatial variation is the source of buoyant potential energy that solely supplies the turbulent kinetic energy; in the model of Overholt \& Pope (1996), the velocity field is forced externally. Second, the active scalar precludes a straightforward extension of the Overholt \& Pope (1996) model for sustaining a passive scalar gradient because the resulting equations governing active scalar fluctuations for large density variations, that is without invoking the Boussinesq approximation, can no longer be simulated in a triply periodic domain. We adapt the fringe-region technique (Bertolotti et al. 1992) to our problem to overcome this difficulty.

Employing the Boussinesq assumption, Batchelor, Canuto \& Chasnov (1992) studied the buoyancy-driven turbulent mixing of an active scalar in a triply periodic domain. Later, Sandoval (1995) and Livescu \& Ristorcelli (2007, 2008) performed similar computations, generalizing to higher density ratios, and without using the Boussinesq assumption. All of these flows were initialized with blobs of unmixed fluid and allowed to decay as the initial potential energy is converted to kinetic energy, which drives the turbulent mixing, before it is finally dissipated by diffusion. Like Sandoval (1995), we presently compute the turbulent mixing of a moderately high- $R$ incompressible binary fluid mixture within a triply periodic domain, but we additionally use a fringe region to sustain an unstable density gradient (heavy fluid on top of light fluid) to produce a statistically stationary flow.

Perhaps the most widely used model to study buoyancy-driven turbulent mixing is the Rayleigh-Taylor instability (e.g. Cook \& Dimotakis 2001; Cook, Cabot \& Miller 2004; Cabot \& Cook 2006; Mueschke \& Schilling 2009), where an initial perturbed interface separating unmixed heavy fluid on top of light fluid is accelerated towards the light fluid, resulting in a growing turbulent mixing layer. RayleighTaylor instability is a statistically evolving flow, requiring expensive computational resources (e.g. Cabot \& Cook 2006) to capture late-time asymptotic self-similar statistics. Our present simulations can perhaps be viewed as a model for the latetime Rayleigh-Taylor instability deep within the interior of the turbulent mixing zone, where the slowly evolving fine-scale turbulence is informed of the far-field boundary conditions only through the unstable density gradient. The analogy is incomplete, however, as a statistically evolving flow is fundamentally different from a statistically stationary flow. Two other flows that are related in this same way 
are forced isotropic-homogeneous turbulence and decaying isotropic-homogeneous turbulence.

A somewhat related flow is the closed-vessel experiment of Krawczynski et al. (2006), where passive scalar mixing is achieved by a continual injection of unmixed fluids from a series of impinging jets, resulting in a statistically stationary homogeneous isotropic turbulent flow. In the present simulations, the role of the jets is played by the fringe region, where unmixed fluids are continually introduced into the domain. Again, we consider a dynamically active scalar and unlike the jets in the experiment, the fringe region is not a source of momentum.

This paper is organized as follows. The governing equations with source terms and the variable-density incompressible fluid model are introduced in $\S 2.1$. We then determine the restrictions on the source terms when solving these equations in a triply periodic domain $(\S 2.2)$. In $\S 2.3$, we introduce our adaptation of the fringeregion technique, and then prescribe a condition on the external pressure gradient in $\S 2.4$. A new method for solving the governing equations that guarantees discrete mass conservation, regardless of iteration errors, is described in $\S 3.1$. The numerical discretization is briefly described in $\$ 3.2$ with the further details provided in the Appendix. We present some direct numerical simulation (DNS) results, including profiles of integral quantities, comparisons of spectra with the Rayleigh-Taylor instability spectra from the DNS data of Cabot \& Cook (2006) and mole fraction probability density functions in $\S 4$. In $\S 5$, we demonstrate the usefulness of the present flow model in the context of developing a subgrid-scale (SGS) active scalar flux model for large-eddy simulations (LES). Finally, we perform LES of the present flow model and present detailed comparisons of spectra with DNS, including subgrid-extended spectra, in $\S 6$ before concluding in $\S 7$.

\section{Problem description}

\subsection{Governing equations}

The species mass conservation equation and the Navier-Stokes equations govern the flow of a binary fluid mixture (see Sandoval 1995; Cook \& Dimotakis 2001; Livescu \& Ristorcelli 2007):

$$
\begin{gathered}
\frac{\partial}{\partial t}\left(\rho Y_{1}\right)+\frac{\partial}{\partial x_{j}}\left(\rho Y_{1} u_{j}-\mathscr{D} \rho \frac{\partial Y_{1}}{\partial x_{j}}\right)=\omega_{1}, \\
\frac{\partial \rho}{\partial t}+\frac{\partial}{\partial x_{j}}\left(\rho u_{j}\right)=0, \\
\frac{\partial}{\partial t}\left(\rho u_{i}\right)+\frac{\partial}{\partial x_{j}}\left(\rho u_{i} u_{j}+p \delta_{i j}-\tau_{i j}\right)=-\Gamma_{i}-\rho g \delta_{i 3}, \\
\tau_{i j}=\mu\left(\frac{\partial u_{i}}{\partial x_{j}}+\frac{\partial u_{j}}{\partial x_{i}}-\frac{2}{3} \delta_{i j} \frac{\partial u_{k}}{\partial x_{k}}\right),
\end{gathered}
$$

where $\rho$ is the density; $u_{i}$ is the velocity; $p$ is the pressure; $\Gamma_{i}(t)$ is the uniform pressure gradient; $g$ is the magnitude of the gravitational acceleration that points in the $-z$ direction; $\tau_{i j}$ is the Newtonian viscous stress tensor; $\omega_{1}(\boldsymbol{x}, t)$ is the source of fluid $1 ; x_{i}$ is the spatial coordinate; $t$ is the time; and $Y_{1}$ is the mass fraction of fluid 1. We use the notations, $(x, y, z)=\left(x_{1}, x_{2}, x_{3}\right)$ and $(u, v, w)=\left(u_{1}, u_{2}, u_{3}\right)$, interchangeably. The mass fraction of fluid 2 is obtained from $Y_{1}+Y_{2}=1$; its evolution 
equation needs not be computed separately. Observe, from the right-hand side of (2.2), that there is no pointwise net source of mass, and so the source of fluid 1 must equal the sink of fluid $2, \omega_{1}(\boldsymbol{x}, t)+\omega_{2}(\boldsymbol{x}, t)=0$. The non-dimensional parameters that characterize the present flow are the Reynolds, Schmidt and Froude numbers, defined as

$$
R e \equiv \rho_{0} U \ell / \mu, \quad S c \equiv \mu /\left(\rho_{0} \mathscr{D}\right), \quad F r^{2} \equiv U^{2} /(g \ell),
$$

where $\rho_{0}$ is the density scale; $U$ is the velocity scale; $\ell$ is the length scale; $\mu=\mu_{1}=\mu_{2}$ is the constant matched dynamic viscosity for both fluids; and $\mathscr{D}$ is the Fickian diffusion coefficient.

Density variation arises purely from variation in the local fluid composition. The relevant equation of state is then (Sandoval 1995)

$$
\frac{1}{\rho(\boldsymbol{x}, t)}=\frac{Y_{1}(\boldsymbol{x}, t)}{\rho_{1}}+\frac{Y_{2}(\boldsymbol{x}, t)}{\rho_{2}}=Y_{1}(\boldsymbol{x}, t)\left(\frac{1}{\rho_{1}}-\frac{1}{\rho_{2}}\right)+\frac{1}{\rho_{2}},
$$

where $\rho_{1}$ and $\rho_{2}$ are the constant microscopic densities of their respective fluids. We fix the density scale $\rho_{0}=\left(\rho_{1}+\rho_{2}\right) / 2$ so that

$$
\frac{\rho_{1}}{\rho_{0}}=1-A \quad \text { and } \quad \frac{\rho_{2}}{\rho_{0}}=1+A, \quad \text { where } \quad A \equiv \frac{\rho_{2}-\rho_{1}}{\rho_{2}+\rho_{1}}=\frac{R-1}{R+1}>0,
$$

the Atwood number. Next, fix the velocity scale

$$
U=(A g \ell)^{1 / 2} \Rightarrow R e=\rho_{0}(A g \ell)^{1 / 2} \ell / \mu, \quad F r^{2}=A,
$$

making $R e, S c$ and $A$ the three independent parameters for this flow. Presently, $S c=1$; we then perform a parametric study in the $(R e, A)$ space.

We eliminate $Y_{1}$ by combining (2.1) and (2.5), and then using (2.2) to get

$$
\frac{\partial u_{j}}{\partial x_{j}}=-\mathscr{D} \frac{\partial}{\partial x_{j}}\left(\frac{1}{\rho} \frac{\partial \rho}{\partial x_{j}}\right)-\omega_{s}, \quad \text { where } \omega_{s} \equiv\left(\frac{1}{\rho_{2}}-\frac{1}{\rho_{1}}\right) \omega_{1},
$$

in contrast to constant density flows, where $\partial u_{j} / \partial x_{j}=0$. We combine (2.7) and (2.2) to write

$$
\frac{\partial s}{\partial t}+u_{j} \frac{\partial s}{\partial x_{j}}=\mathscr{D} \frac{\partial^{2} s}{\partial x_{j}^{2}}+\omega_{s}
$$

where $s \equiv \log \left(\rho / \rho_{0}\right)$. We will use (2.8) as an alternative evolution equation for $\rho$.

\subsection{Consequences of periodicity}

We wish to compute a non-trivial solution to the governing equations in a periodic domain. Given this constraint, we will now determine how to obtain a flow that is statistically stationary by choosing $\omega_{1}$ in (2.1) or equivalently $\omega_{s}$ in (2.7). Denote the volume average by []$_{x}$, then periodicity implies $\left[\partial() / \partial x_{j}\right]_{x}=0$. From $(2.1)$ and (2.2),

$$
\frac{\partial}{\partial t}\left[\rho Y_{1}\right]_{x}=\left[\omega_{1}\right]_{x}, \quad \frac{\partial[\rho]_{x}}{\partial t}=0 .
$$

Without loss of generality, set $[\rho]_{x}=\rho_{0}$. We rearrange (2.5) and (2.6), and then volume 
average to get

$$
\left[\rho Y_{1}\right]_{x}=\left(1-[\rho]_{x} / \rho_{2}\right) /\left(1 / \rho_{1}-1 / \rho_{2}\right)=\rho_{1} / 2=\rho_{0}(1-A) / 2,
$$

a constant; likewise, $\left[\rho Y_{2}\right]_{x}=\rho_{0}(1+A) / 2$. Since $\left[\rho Y_{1}\right]_{x}$ and $\left[\rho Y_{2}\right]_{x}$ are constants, (2.9) implies that

$$
\left[\omega_{1}\right]_{x}=\left[\omega_{2}\right]_{x}=\left[\omega_{s}\right]_{x}=0
$$

at every instant of time.

Decomposing $\rho=[\rho]_{x}+\rho^{\dagger}$, we can obtain the evolution equation for $\rho^{\dagger 2}$ from (2.2):

$$
\frac{\partial \rho^{\dagger 2}}{\partial t}+\frac{\partial}{\partial x_{j}}\left(u_{j} \rho^{\dagger 2}\right)+\left(\rho^{2}-[\rho]_{x}^{2}\right) \frac{\partial u_{j}}{\partial x_{j}}=0 .
$$

We now use (2.7) to calculate

$$
\rho^{2} \frac{\partial u_{j}}{\partial x_{j}}=-\mathscr{D}\left(\frac{\partial^{2}}{\partial x_{j}^{2}}\left(\frac{1}{2} \rho^{2}\right)-2\left(\frac{\partial \rho}{\partial x_{j}}\right)^{2}\right)-\rho^{2} \omega_{s},
$$

which we then combine with the volume average of (2.11) to obtain the equation governing the density fluctuation variance:

$$
\frac{\partial\left[\rho^{\dagger 2}\right]_{x}}{\partial t}=-2 \mathscr{D}\left[\left(\frac{\partial \rho^{\dagger}}{\partial x_{j}}\right)^{2}\right]_{x}+\left[\rho^{2} \omega_{s}\right]_{x} .
$$

Denote the long-time average by []$_{t}$, then $[\partial() / \partial t]_{t}=0$ for any statistically stationary quantity. Time averaging (2.12),

$$
2 \mathscr{D}\left[\left[\left(\partial \rho^{\dagger} / \partial x_{j}\right)^{2}\right]_{x}\right]_{t}=\left[\left[\rho^{2} \omega_{s}\right]_{x}\right]_{t}>0 .
$$

We choose $\omega_{s}(\boldsymbol{x}, t)=0$ except in a region called the fringe. Then (2.13) says that, over time, the source of unmixed fluids, introduced in the fringe, necessarily balances the mixing occurring outside the fringe, resulting in a statistically stationary flow.

\subsection{Fringe-region forcing}

To date, we have not constrained $\omega_{1}(\boldsymbol{x}, t)$ and $\omega_{2}(\boldsymbol{x}, t)$ to produce a non-trivial statistically stationary flow; we now address this issue. A source of unmixed fluids in unstable configuration (heavy fluid on top of light fluid) is required for buoyancy forces to drive the turbulent mixing process. In Rayleigh-Taylor turbulence, the infinite reservoirs of unmixed fluid supply the mixing zone, but the flow is not stationary owing to the growing height of the mixing zone. The kind of stationary flow that we envision presently has similarities with the partially stirred reactor of Krawczynski et al. (2006), which was used to study passive scalar mixing by jet-driven turbulence in a closed vessel. In our case, the scalar is active and the turbulence is driven by buoyancy (not momentum).

Our goal is to simulate a turbulent mixing flow in a triply periodic domain. In the absence of any external forcing, the flow eventually decays, which is the flow computed by Livescu \& Ristorcelli (2007). The present approach is to approximate the mixing chamber by using the fringe-region technique (e.g. Bertolotti et al. 1992; Nordström et al. 1999). A natural choice is to apply the technique directly to the source term $\omega_{1}$ in $(2.1)$ :

$$
\omega_{1}(\boldsymbol{x}, t)=\Lambda_{1} \lambda_{1}(\boldsymbol{x}) \rho_{1} Y_{2}(\boldsymbol{x}, t)-\Lambda_{2} \lambda_{2}(\boldsymbol{x}) \rho_{2} Y_{1}(\boldsymbol{x}, t),
$$



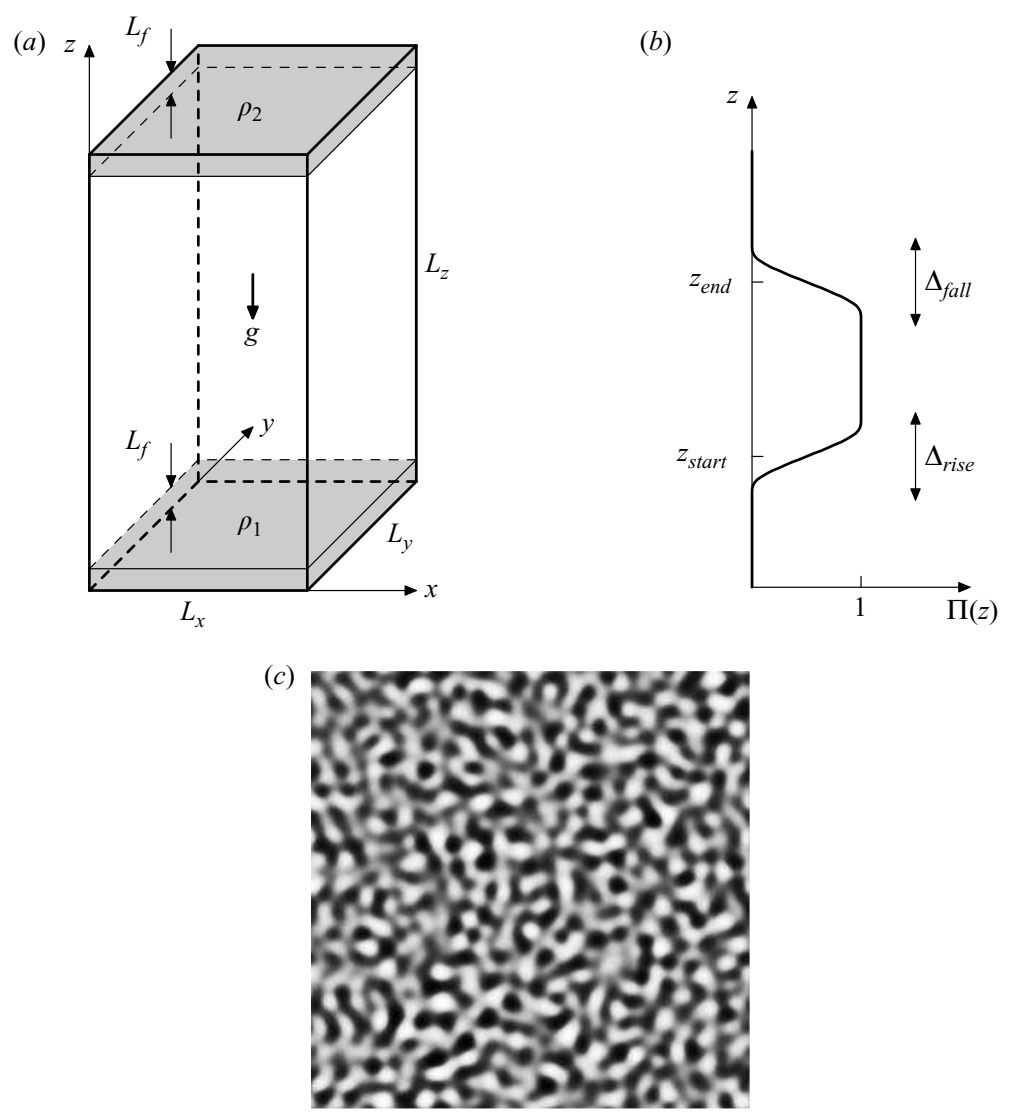

FIGURE 1. (a) Triply periodic flow domain showing the shaded fringe regions that supply the flow with unmixed fluids, heavy above light $\left(\rho_{2}>\rho_{1}\right)$. (b) Features of the smooth function $\Pi(z)$, $(2.16 a)$, used to locate the fringe region. $(c)$ Horizontal slice of the planar indicator function $\xi_{1}(x, y)$, constructed from applying the Gaussian spectral filter to a physical i.i.d. random field of $N(0,1)$. The filter is centred on wavenumber $k_{0} \ell$ corresponding to wavelength $\lambda_{0} / \ell=2 \pi / 16$ and the box shown has dimensions $2 \pi \ell \times 2 \pi \ell$.

or equivalently, using (2.5) and (2.7),

$$
\omega_{s}(\boldsymbol{x}, t)=\Lambda_{1} \lambda_{1}(\boldsymbol{x})\left(\rho_{1} / \rho(\boldsymbol{x}, t)-1\right)+\Lambda_{2} \lambda_{2}(\boldsymbol{x})\left(\rho_{2} / \rho(\boldsymbol{x}, t)-1\right),
$$

where $0 \leqslant \lambda_{1}, \lambda_{2} \leqslant 1$ are the smooth fringe indicator functions (1 inside the fringe region, 0 outside the fringe region) corresponding to the respective fluid sources. Momentarily setting $\left(\lambda_{1}, \lambda_{2}\right)=(1,0)$ in $(2.14 a)$, observe that the rate at which the light fluid is introduced in the flow is proportional to its microscopic density $\rho_{1}$ and its mass fraction deficit $Y_{2}=1-Y_{1}$. A similar statement can be made for the heavy fluid source. The indicator functions are defined by

$$
\begin{aligned}
& \lambda_{1}(\boldsymbol{x})=\xi_{1}(x, y)\left(\Pi\left(z ; 0,0+L_{f}\right)+\Pi\left(z ; L_{z}, L_{z}+L_{f}\right)\right), \\
& \lambda_{2}(\boldsymbol{x})=\xi_{2}(x, y)\left(\Pi\left(z ; 0-L_{f}, 0\right)+\Pi\left(z ; L_{z}-L_{f}, L_{z}\right)\right),
\end{aligned}
$$

where $L_{z}$ is the height of the periodic domain, shown in figure $1(a) ; L_{f} / \ell=2 \pi / 10$, the height of the fringe region; $0 \leqslant \xi_{1}, \xi_{2} \leqslant 1$ are planar indicator functions to be defined; and $\Pi$ is a top-hat function constructed from smooth step functions $S$ (see 
figure $1 b$ )

$$
\begin{aligned}
\Pi\left(z ; z_{\text {start }}, z_{\text {end }}\right) & =S\left(\frac{z-z_{\text {start }}}{\Delta_{\text {rise }}}+\frac{1}{2}\right)-S\left(\frac{z-z_{\text {end }}}{\Delta_{\text {fall }}}+\frac{1}{2}\right), \\
S(z) & = \begin{cases}0, & z \leqslant 0, \\
1 /\left(1+\exp \left(\frac{1}{z-1}+\frac{1}{z}\right)\right), & 0<z<1, \\
1, & z \geqslant 1 .\end{cases}
\end{aligned}
$$

We choose transition widths $\Delta_{\text {rise }}=\Delta_{\text {fall }}=6 \Delta_{z}$, where $\Delta_{z}$ is the computational cell height. The indicator functions $\lambda_{1}(x)$ and $\lambda_{2}(x)$ in (2.15) are chosen so that heavy fluid is introduced at the top of the flow domain and light fluid is introduced at the bottom of the domain. We use $\Pi$ twice in each of (2.15) to preserve vertical periodicity.

The planar indicator functions are constrained to have zero mean: $\left\langle\xi_{1}(x, y)\right\rangle=\left\langle\xi_{2}(x, y)\right\rangle=0$, where \langle\rangle denotes the $x y$-plane average. They are constructed in a manner similar to the construction of the perturbation field used by Cook et al. (2004). Briefly, independent and identically distributed (i.i.d.) normal random variables with zero mean and unit variance, $N(0,1)$, are assigned to each $(x, y)$ grid point. We transform this field to Fourier space and apply a Gaussian filter centred on wavenumber $k_{0} \ell=16$ with standard deviation $\sigma_{k} \ell=4$. The resulting field is transformed back to physical space with value $\zeta(x, y)$ and steepened with the function

$$
\xi_{1}(x, y)=1 / 2+\arctan \left(\pi \zeta(x, y) /\left(3 \sigma_{\zeta}\right)\right) / \pi
$$

where $\sigma_{\zeta}$ is the standard deviation of $\zeta$. A contour plot of $\xi_{1}$ is shown in figure 1(c).

Substituting (2.14b) in (2.8) and rearranging, we obtain the simple balance between the source term and the unsteady term in the fringe:

$$
\frac{\partial \rho}{\partial t}=\left\{\begin{array}{lll}
\Lambda_{1}\left(\rho_{1}-\rho\right) & \text { if } & \left(\lambda_{1}, \lambda_{2}\right)=(1,0) \\
\Lambda_{2}\left(\rho_{2}-\rho\right) & \text { if } \quad\left(\lambda_{1}, \lambda_{2}\right)=(0,1)
\end{array}\right\}+\text { other terms }
$$

Observe that $\omega_{s}$ is designed to force $\rho(\boldsymbol{x}, t)$ following a fluid particle to track $\rho_{1}$ (or $\rho_{2}$ ) at the rate $\Lambda_{1}$ (or $\Lambda_{2}$ ). The fringe-region rates, $\Lambda_{1}$ and $\Lambda_{2}$, are similar to the Damköhler number used in chemical reactions - they set the strength of the fluid sources relative to the flow. They can also be interpreted as inverse time constants of first-order systems, clearly seen in structure of (2.17). They are not independent; recall from (2.10) that

$$
\Lambda_{1}\left[\lambda_{1}(\boldsymbol{x}, t)\left(\rho_{1} / \rho-1\right)\right]_{x}+\Lambda_{2}\left[\lambda_{2}(\boldsymbol{x}, t)\left(\rho_{2} / \rho-1\right)\right]_{x}=0 .
$$

It remains to fix the upper limit:

$$
\Lambda=\max \left\{\Lambda_{1}, \Lambda_{2}\right\} .
$$

We use an order-of-magnitude argument to choose $\Lambda$. Since the fringe introduces unmixed fluids with densities $\rho_{1}$ and $\rho_{2}$ in a layer of width $L_{f}$ subjected to gravity $g$, its characteristic velocity scale is $U_{f}=\left(A g L_{f}\right)^{1 / 2}$. The time it takes for a fluid particle to transit through the fringe is $T_{f}=L_{f} / U_{f}=\left(L_{f} / A g\right)^{1 / 2}$. We then choose $\Lambda(\ell / A g)^{1 / 2}=10$, which is much larger than the transit rate, $T_{f}^{-1}(\ell / A g)^{1 / 2}=\left(\ell / L_{f}\right)^{1 / 2}=(10 / 2 \pi)^{1 / 2} \approx 1.26$, a source rate high enough, relative to the flow, in order for $\rho(\boldsymbol{x}, t)$ to take on the desired values $\rho_{1}$ or $\rho_{2}$. 


\subsection{Mean pressure gradient}

A model is required for $\Gamma_{i}(t)$, the externally imposed spatially uniform pressure gradient. In Rayleigh-Taylor turbulence, the far-field quiescent boundary conditions determine $\Gamma_{i}$ felt in the turbulent mixing zone. In a triply periodic domain where such far-field boundary conditions cannot be directly imposed, we model $\Gamma_{i}$ by requiring that $\left\langle\partial u_{i} / \partial t\right\rangle_{m}=0$, where \langle\rangle$_{m}$ denotes the $x y$-plane average taken at the mid-plane $z=L_{z} / 2$. As noted by Livescu \& Ristorcelli (2007), $\left\langle\partial u_{i} / \partial t\right\rangle_{m} \approx 0$ in the Rayleigh-Taylor turbulent mixing zone; they considered a similar model by choosing $\left[\partial u_{i} / \partial t\right]_{x}=0$. For definiteness, we choose $\left\langle u_{i}\right\rangle_{m}=0$. Alternatively, $\Gamma_{i}$ can be determined from the volume average of (2.3),

$$
\frac{\partial}{\partial t}\left[\rho u_{i}\right]_{x}=-\Gamma_{i}(t)-[\rho]_{x} g \delta_{i 3} .
$$

Upon taking the long-time average, the $\partial / \partial t$ term vanishes, and we obtain $\left[\Gamma_{i}\right]_{t}=$ $-\left[[\rho]_{x}\right]_{t} g \delta_{i 3}=-\rho_{0} g \delta_{i 3}$. That is, over time, $\Gamma_{i}(t)$ fluctuates about a known steady state. We use this result to check the internal consistency of our code.

\section{Solution method}

\subsection{Alternative Lagrange multiplier to pressure}

In incompressible flows, a constraint on the velocity divergence has to be satisfied at all times. For variable-density flows, the constraint is (2.7), while for constantdensity flows, the constraint is $\partial u_{j} / \partial x_{j}=0$. This is enforced by treating $p$ as a Lagrange multiplier. The elliptic equation for $p$ is obtained by taking the divergence of (2.3), then enforcing the constraint (2.7). In constant-density flows, this results in a constant-coefficient Poisson equation for $p$, which is readily solved. The non-constant $1 / \rho$ factor in variable-density flows presents an additional complication.

This issue appears in a variety of forms in the literature and cannot be circumvented. Sandoval (1995) and Cook \& Dimotakis (2001), for example, take the divergence of (2.3), resulting in a constant-coefficient Poisson equation for $p$, but use what amounts to a lower-order extrapolation for $\partial u_{i} / \partial t$, reducing the overall accuracy of the temporal discretization. Consequently, mass conservation in the form of (2.7) is never satisfied instantaneously. The advantage to their approach is that no iteration is required. Another approach to this issue is proposed by Livescu \& Ristorcelli (2007), who derive an exact nonlinear equation for $p$ ((A 15) in that paper) that requires an iterative solution method but eliminates temporal discretization errors. However, it remains that (2.7) cannot be discretely satisfied owing to the inevitable finite spatial resolution, even if infinite iterations were possible.

Our approach eliminates some, but not all, of these difficulties. For clarity, continuous differential operators will be used in the exposition but the method applies directly to their discrete counterparts. First, expand (2.3) and use (2.2) to get

$$
\frac{\partial u_{i}}{\partial t}=-\frac{1}{\rho}\left(\frac{\partial p}{\partial x_{i}}+\Gamma_{i}\right)-u_{j} \frac{\partial u_{i}}{\partial x_{j}}+\frac{1}{\rho} \frac{\partial \tau_{i j}}{\partial x_{j}}-g \delta_{i 3} .
$$

The idea is to use the following Helmholtz-Hodge decomposition (see Tong et al. 2003):

$$
\frac{1}{\rho}\left(\frac{\partial p}{\partial x_{i}}+\Gamma_{i}\right)=\frac{\partial \phi}{\partial x_{i}}+h_{i}+f_{i}, \quad h_{i}=\epsilon_{i j k} \frac{\partial \psi_{k}}{\partial x_{j}} .
$$


That is, instead of $\left(p, \Gamma_{i}\right)$, we use the alternative Lagrange multipliers $\left(\phi, \psi_{i}, f_{i}\right)$, where $\phi$ is the scalar potential, $\psi_{i}$ is the vector potential and $f_{i}$ is the harmonic component. With periodic boundary conditions, the harmonic component $f_{i}(t)$ is spatially uniform. Put (3.2) back into (3.1) to obtain

$$
\frac{\partial u_{i}}{\partial t}=\frac{\partial \phi}{\partial x_{i}}+h_{i}+f_{i}+H_{i}
$$

where $H_{i}$ contains the remaining terms in (3.1). Taking the divergence of (3.3) immediately gives a constant-coefficient Poisson equation for $\phi$ with a known righthand side:

$$
\frac{\partial^{2} \phi}{\partial x_{j}^{2}}=\frac{\partial}{\partial t}\left(\frac{\partial u_{j}}{\partial x_{j}}\right)-\frac{\partial H_{j}}{\partial x_{j}},
$$

where $\partial u_{j} / \partial x_{j}$ is found from (2.7). The solution to the discrete form of this equation is straightforward. Next, $f_{i}$ is found from taking the $z=L_{z} / 2$ plane average of (3.3) and applying the model boundary condition $(\$ 2.4):\left\langle\partial u_{i} / \partial t\right\rangle_{m}=0$ so

$$
f_{i}(t)=-\left\langle\frac{\partial \phi}{\partial x_{i}}+h_{i}+H_{i}\right\rangle_{m} .
$$

To solve for $h_{i}$, we first multiply (3.2) by $\rho$, then take the curl, giving a zero left-hand side:

$$
0=\epsilon_{i j k} \frac{\partial}{\partial x_{j}}\left(\rho\left(\frac{\partial \phi}{\partial x_{k}}+h_{k}+f_{k}\right)\right) .
$$

This is essentially the equation for $h_{i}$. We can simplify this further by splitting $\rho=[\rho]_{x}+\rho^{\dagger}$ and using the gauge $\partial \psi_{j} / \partial x_{j}=0$,

$$
\frac{\partial^{2} \psi_{i}}{\partial x_{j}^{2}}=\epsilon_{i j k} \frac{\partial}{\partial x_{j}}\left(\frac{\rho^{\dagger}}{[\rho]_{x}}\left(\frac{\partial \phi}{\partial x_{k}}+h_{k}+f_{k}\right)\right) .
$$

Finally, take the curl again to obtain the nonlinear equation

$$
\frac{\partial^{2} h_{i}}{\partial x_{j}^{2}}=\frac{\partial}{\partial x_{i}} \frac{\partial B_{j}}{\partial x_{j}}-\frac{\partial^{2} B_{i}}{\partial x_{j}^{2}}, \quad B_{i}=\frac{\rho^{\dagger}}{[\rho]_{x}}\left(\frac{\partial \phi}{\partial x_{i}}+h_{i}+f_{i}\right) .
$$

We solve this by iterating: use the current $h_{i}^{(n)}$ in the right-hand side $B_{i}^{(n+1)}$ of the Poisson equation for the next $h_{i}^{(n+1)}$. If the density is constant, $\rho^{\dagger}=0 \Rightarrow B_{i}=0 \Rightarrow$ $h_{i}=0$ to recover $\left(p, \Gamma_{i}\right)=\left(\phi, f_{i}\right)$, verifiable from (3.4) and (3.2).

Using (3.2) allows the exact satisfaction of mass conservation (2.1) at every time instant and up to the machine precision of the spatial discretization, regardless of iteration errors in (3.4). This is because the part of the Lagrange multiplier $(1 / \rho)\left(\partial p / \partial x_{i}\right)$ that plays the role of mass conservation is completely encapsulated by its curl-free component $\partial \phi / \partial x_{i}$. All errors from this method are isolated to iteration residuals in $h_{i}$. In the vorticity equation, its curl, $\epsilon_{i j k} \partial h_{k} / \partial x_{j}$, is the baroclinic source of vorticity. In physical terms, the present approach eliminates mass conservation errors but incurs errors on baroclinic vorticity. However, the vorticity equation is always subject to temporal discretization errors. A similar approach for the constantdensity incompressible equations is taken by Chang, Giraldo \& Perot (2002). Using $h_{i}$ from the previous time step as a first guess, we presently control this error by iterating until $\left\|h_{i}^{(n+1)}-h_{i}^{(n)}\right\| /\left\|h_{i}^{(n)}\right\|<10^{-2}$, where \|\| denotes the $L_{2}$-norm. In theory, the convergence rate could be sensitive to $A$ since $B_{i}$ in (3.4) depends explicitly on the density fluctuations, $\rho^{\dagger} / \rho_{0}=O(A)$, but for the present cases at 
$A=1 / 2,3 / 4,1-2$ iterations give convergence using the same Courant-FriedrichsLevy number, $\mathrm{CFL}=0.7$ (defined in the Appendix). If the accurate time integration of the divergence-free component $h_{i}$ is not of primary concern, one can always eliminate the iteration step by simply using the first guess $h_{i}$ from the previous time step. In this case, the cost of the scheme is similar to the one in Cook \& Dimotakis (2001), but, presently, discrete mass conservation is still satisfied.

Since $p$ and $\Gamma_{i}$ have been replaced by $\phi, \psi_{i}$ and $f_{i}$, they are not required for the time integration of the governing equations. If required for diagnostics, they are readily calculated from

$$
\frac{\partial^{2} p}{\partial x_{j}^{2}}=\frac{\partial}{\partial x_{j}}\left(\rho\left(\frac{\partial \phi}{\partial x_{j}}+h_{j}+f_{j}\right)\right), \quad \Gamma_{i}(t)=\left[\rho\left(\frac{\partial \phi}{\partial x_{i}}+h_{i}+f_{i}\right)\right]_{x} .
$$

We remark that the satisfaction of discrete mass conservation is only one of many ways to assess the 'goodness' of a solution. However, anecdotal evidence in the literature suggests that discrete mass conservation is important for numerical stability. Sandoval (1995), for example, reports numerical instability in a version of his code that uses an extrapolation of $\partial \rho / \partial t$ in flows with large density ratios. Using the present discretization, no such numerical instability was found, even when $R=7$. A study exploring a direct link between discrete mass conservation and numerical stability is beyond the scope of this work.

\subsection{Numerical discretization}

The governing equations, in the form (2.7), (2.8) and (3.1), are advanced using the lowstorage semi-implicit Runge-Kutta method of Spalart, Moser \& Rogers (1991). The spatial discretization employs the Fourier pseudospectral method: compute products and nonlinear terms in physical space, then use the 2/3-rule for dealiasing. The maximum dealiased wavenumber is then $(2 / 3)(\pi / \Delta)$, where $\Delta$ is the grid size. We provide further details in the Appendix.

\subsection{Code validation}

As validation of our code, we reproduce the case 3Base of Livescu \& Ristorcelli (2007) from three independent but statistically identical initial conditions (see figure 2). This is readily achieved by setting $\omega_{s}=0$ in (2.7) and (2.8), choosing $\Gamma_{i}(t)$ so that $\left[u_{i}\right]_{x}=0$ (see $\S 2.4$ ) and initializing the flow as random blobs of pure fluids in a cube of size $2 \pi \ell$, a procedure documented by Livescu \& Ristorcelli (2007). There is some statistical spread in the present initial conditions: the initial (volume-averaged) integral length scale is $0.3542-0.3550 \ell$, and the initial density fluctuation variance $0.2248-0.2252 \rho_{0}^{2}$. Livescu \& Ristorcelli (2007) reported these numbers as $0.3525 \ell$ and $0.22 \rho_{0}^{2}$, respectively.

Physically (see figure 2), the flow starts at $t=0$ from rest, $\left[\rho u_{i} u_{i}\right]_{x}=0$, driven by potential energy stored in unmixed blobs of different densities with variance $\left[\rho^{\dagger 2}\right]_{x}^{1 / 2} / \rho_{0} \approx 0.22$. As the fluid mixes, the density variance decreases while the kinetic energy increases to peak at $t(A g / \ell)^{1 / 2} \approx 2$ before the mixture eventually decays to a homogenized and quiescent state at large times.

\section{DNS results and discussion}

The DNS parameters are given in table 1. In each case, the horizontal crosssection is a square, $L_{x}=L_{y}$. To assess sensitivities to the choice of computational 


\begin{tabular}{ccccccccccccccc}
\hline Key & Case & $R e$ & $R e_{\Delta}$ & $S c$ & $A$ & $R$ & $L_{x} / \ell$ & $L_{y} / \ell$ & $L_{z} / \ell$ & $L_{f} / \ell$ & $N_{x}$ & $N_{y}$ & $N_{z}$ & $T_{e}$ \\
$-\cdot-\cdot-$ & $\mathrm{A}$ & 260 & 1 & 1 & $1 / 2$ & 3 & $2 \pi$ & $2 \pi$ & $2 \pi$ & $2 \pi / 10$ & 256 & 256 & 256 & 5.6 \\
$\cdots \cdots \cdots$ & $\mathrm{B}$ & 260 & 1 & 1 & $1 / 2$ & 3 & $4 \pi$ & $4 \pi$ & $2 \pi$ & $2 \pi / 10$ & 512 & 512 & 256 & 3.2 \\
$-\cdots$ & $\mathrm{C}$ & 260 & 1 & 1 & $1 / 2$ & 3 & $2 \pi$ & $2 \pi$ & $4 \pi$ & $2 \pi / 10$ & 256 & 256 & 512 & 5.3 \\
-- & $\mathrm{D}$ & 260 & 1 & 1 & $3 / 4$ & 7 & $2 \pi$ & $2 \pi$ & $4 \pi$ & $2 \pi / 10$ & 256 & 256 & 512 & 8.5 \\
--- & $\mathrm{E}$ & 478 & 1 & 1 & $1 / 2$ & 3 & $2 \pi$ & $2 \pi$ & $4 \pi$ & $2 \pi / 10$ & 384 & 384 & 768 & 5.3 \\
- & $\mathrm{F}$ & 478 & 1 & 1 & $3 / 4$ & 7 & $2 \pi$ & $2 \pi$ & $4 \pi$ & $2 \pi / 10$ & 384 & 384 & 768 & 4.0
\end{tabular}

TABLE 1. DNS parameters. $T_{e}$ is defined in (4.1).
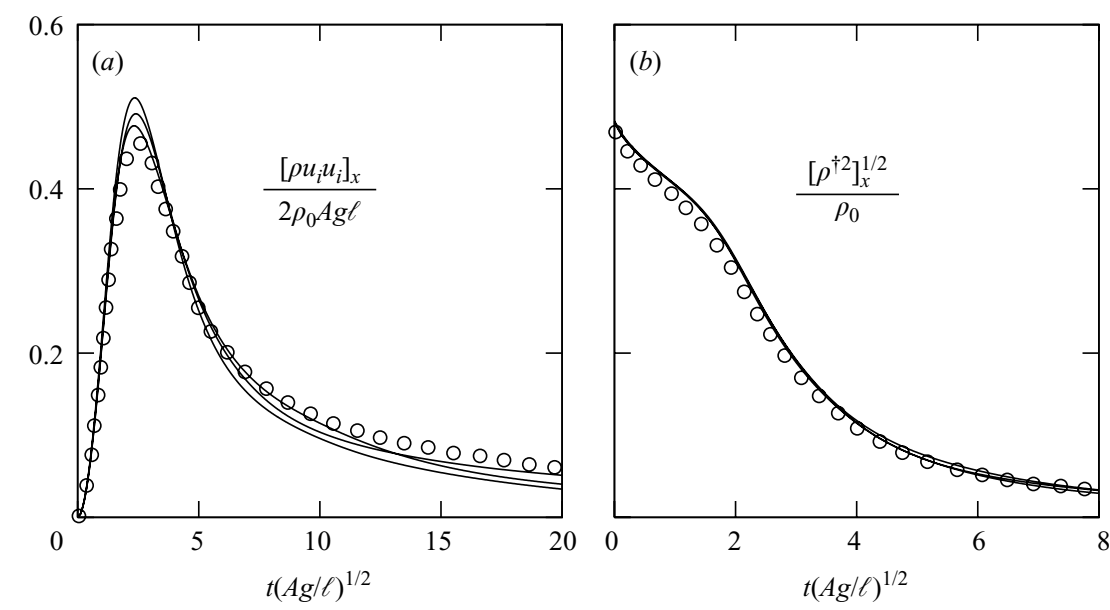

FIGURE 2. The present code shows agreement when validated against a simulation performed by Livescu \& Ristorcelli (2007) (०). Three independent but statistically identical simulations are shown (- $)$.

domain, we perform the set of cases $\mathrm{A}, \mathrm{B}$ and $\mathrm{C}$, which have identical $R e$ and $A$, but different computational domains: case $\mathrm{A}$ in a cube domain $L_{z}=L_{x}$; case $\mathrm{B}$ in a short domain $L_{z}=L_{x} / 2$; and case $\mathrm{C}$ in a tall domain $L_{z}=2 L_{x}$. Cases $\mathrm{C}, \mathrm{D}, \mathrm{E}$ and $\mathrm{F}$ share the same tall domains, but have the four different permutations of $A=\{1 / 2,3 / 4\}$ and $R e=\left\{(256 / 2 \pi)^{3 / 2},(384 / 2 \pi)^{3 / 2}\right\}=\{260,478\}$. The grid Reynolds numbers $\operatorname{Re}_{\Delta} \equiv \rho_{0}(A g \Delta)^{1 / 2} \Delta / \mu=\operatorname{Re}(\Delta / \ell)^{3 / 2}$ are set to unity; the grid is equidimensional, $\Delta=L_{x} / N_{x}=L_{y} / N_{y}=L_{z} / N_{z}$. Simulations are advanced until volume-averaged statistics appear to reach a statistically stationary state at $t=t_{\text {start }}$. Then all statistics are averaged over $T_{e}$ eddy-turnover times,

$$
T_{e} \equiv\left(t_{\text {end }}-t_{\text {start }}\right)\left(\left[u_{i}^{\dagger 2}\right]_{x} / 3\right)^{1 / 2} / L_{x},
$$

listed in table 1. Unless stated otherwise, we will remove time dependence from all statistics to imply time averaging, avoiding cumbersome notation.

We show in figure 3 visualizations of the heavy-fluid mole fraction

$$
X_{2}(\boldsymbol{x}, t) \equiv \frac{\rho(\boldsymbol{x}, t)-\rho_{1}}{\rho_{2}-\rho_{1}} .
$$


(a)

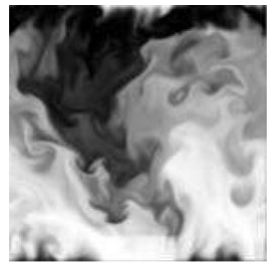

(b)

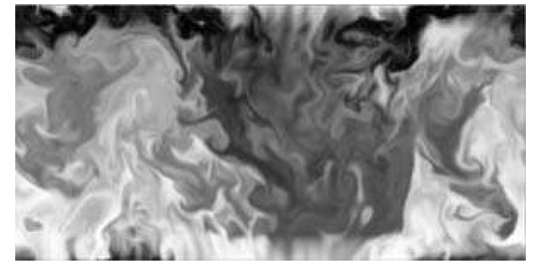

(c)

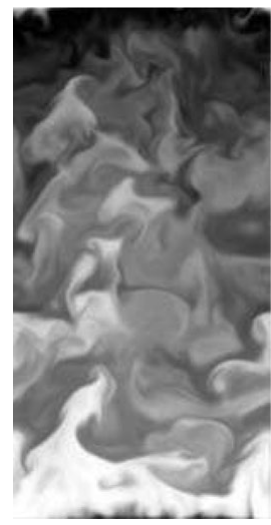

(d)

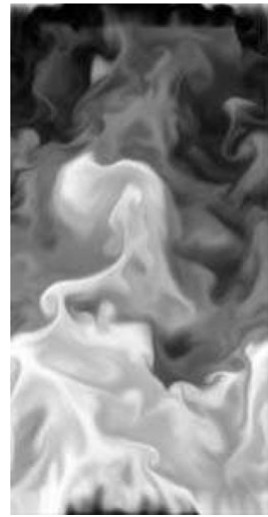

(e)

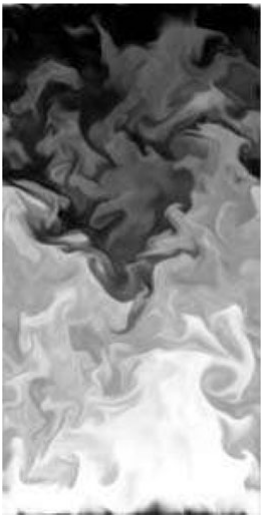

(f)

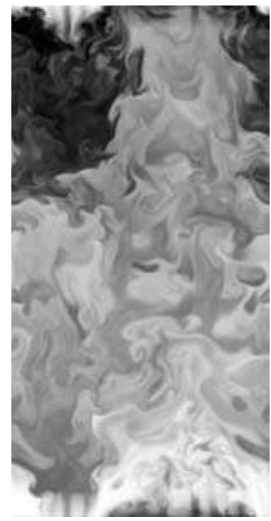

FIGURE 3. Representative $x z$-plane visualizations of $X_{2}$, defined by (4.2). Gravity points downward. The shade varies from light to dark as $X_{2}$ varies from 0 to 1 . See table 1 for simulation parameters. $(a)$ Case $\mathrm{A},(b)$ case $\mathrm{B},(c)$ case $\mathrm{C},(d)$ case $\mathrm{D},(e)$ case $\mathrm{E}$ and $(f)$ case $\mathrm{F}$.

\subsection{Integral and Taylor statistics}

We define the characteristic horizontal wavelength $l_{\rho}$ and integral length scale $L_{\rho}$ of the density fluctuations at an $x y$-plane as

$$
l_{\rho}(z) \equiv 2 \pi L_{\rho}(z) \equiv 2 \pi \frac{\int_{0}^{\infty} E_{\rho \rho}^{2 D}\left(k_{r}\right) / k_{r} \mathrm{~d} k_{r}}{\int_{0}^{\infty} E_{\rho \rho}^{2 D}\left(k_{r}\right) \mathrm{d} k_{r}},
$$

where the planar cospectrum $E_{f g}^{2 D}\left(k_{r}\right)$ of the field variables, $f$ and $g$, is normalized so that

$$
\left\langle f^{\prime} g^{\prime}\right\rangle=\int_{0}^{\infty} E_{f g}^{2 D}\left(k_{r}\right) \mathrm{d} k_{r}
$$

where $k_{r}^{2}=k_{x}^{2}+k_{y}^{2}, f=\langle f\rangle+f^{\prime}$ and $g=\langle g\rangle+g^{\prime}$. The characteristic horizontal wavelength is defined in (4.3) so that a delta-function spectrum, $E_{\rho \rho}^{2 D}=\delta\left(k_{r}-k_{\rho}\right)$, would recover the standard definition $l_{\rho}=2 \pi / k_{\rho}$. We remark that $L_{\rho}$, defined as the integral of the plane-averaged autocorrelation function, differs by a factor of $\pi / 2$ to its the volume-averaged counterpart.

Observe from the profile of $l_{\rho}$ in figure $4(a, d)$ that, regardless of the aspect ratio $L_{z} / L_{x}$ used for the computational domain, the characteristic wavelength of eddies (based on density fluctuations) is given by $l_{\rho} \approx 0.5 L_{x}\left(L_{\rho} \approx 0.08 L_{x}\right)$. This rules out a statistically stationary simulation that is independent of the horizontal box size, an issue also commonly found in forced homogeneous-isotropic turbulence simulations, where the energy-based integral length scale is reported as $\approx 0.15 L_{x}$ (e.g. Overholt $\&$ Pope 1996). This implies that, similar to forced homogeneous-isotropic turbulence 

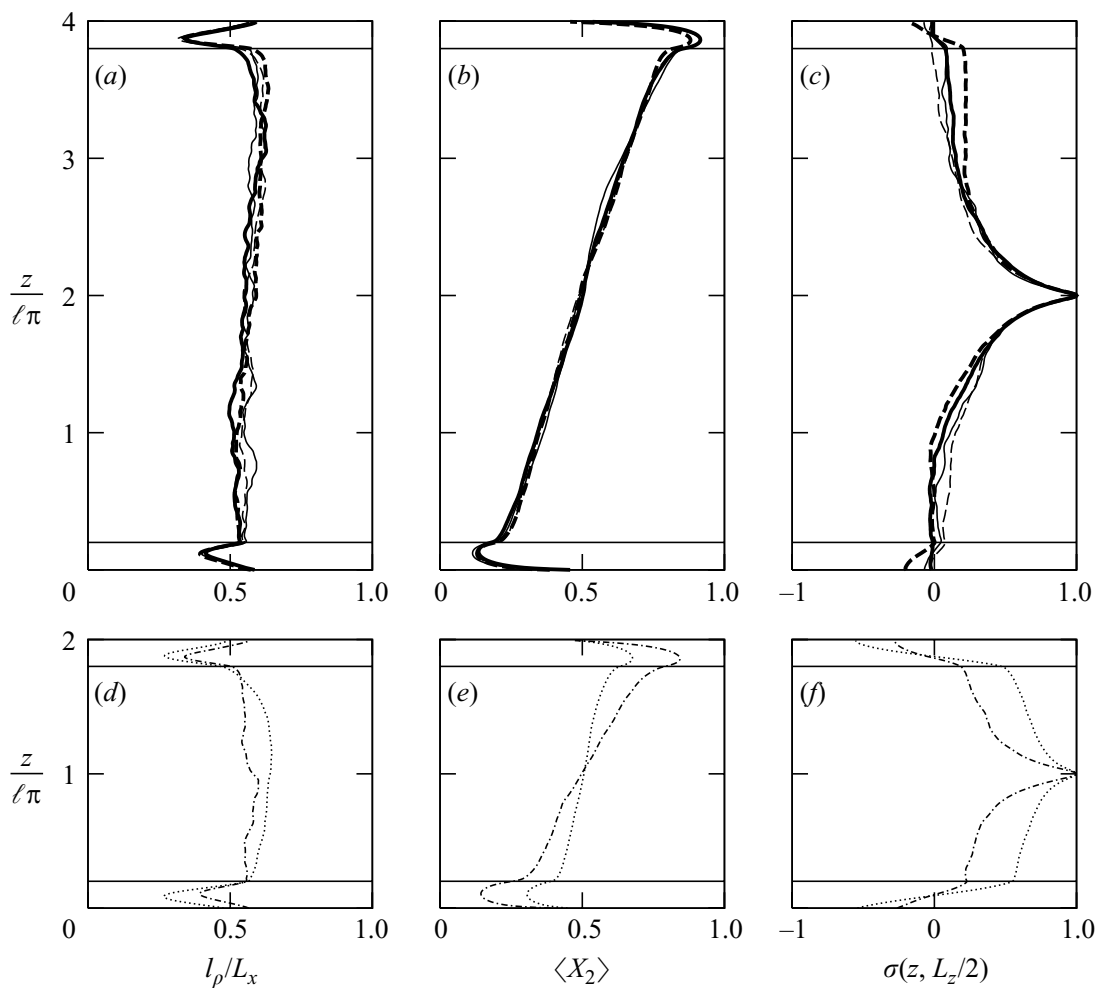

Figure 4. Profiles of the integral quantities $l_{\rho}$ defined by (4.3), $X_{2}$ defined by (4.2) and $\sigma\left(z, L_{z} / 2\right)$ defined by (4.5): $(a-c)$ tall boxes, $L_{z} / \ell=4 \pi ;(d-f)$ short boxes, $L_{z} / \ell=2 \pi$ (see table 1 for key). Horizontal lines indicate fringe-region boundaries.

simulations, the present simulations should be viewed as a model to study only the small scales of buoyancy-driven variable-density turbulent mixing. Since there are no physical length scales in our simulations, the existence of box-filling eddies is hardly surprising. This issue is also relevant in Rayleigh-Taylor instability simulations, where, to preserve the physical relevance of results, simulations are typically stopped before the size of the eddies overwhelm the box (Cook et al. 2004).

The profiles of $\left\langle X_{2}\right\rangle$ for the tall boxes $\left(L_{z}=2 L_{x}\right)$, but with different $R e$ and $A$, collapse (figure $4 b$ ), outside the fringes, onto an approximate line that passes through the point $\left\langle X_{2}\right\rangle=0.5$ at $z=L_{z} / 2$. In the upper fringe, $\left\langle X_{2}\right\rangle<1$ and, conversely, in the lower fringe, $\left\langle X_{2}\right\rangle>0$, implying that the 'unmixing' process in the fringe is not complete, a desirable feature if we do not want the flow to relaminarize; a fringe with infinite source rate $\Lambda$ implies $\left\langle X_{2}\right\rangle=0$ for the lower fringe and $\left\langle X_{2}\right\rangle=1$ for the upper fringe. The influence of $L_{z} / L_{x}$ on the overall slope of $\left\langle X_{2}\right\rangle$ is evident from figure $4(b, e)$ : a shorter box relative to its width reduces the slope of $\left\langle X_{2}\right\rangle$.

To check that the mid-plane statistics are independent of the fringe region, we plot the density fluctuation autocorrelation $\sigma\left(z, L_{z} / 2\right)$, where

$$
\sigma\left(z_{1}, z_{2}\right)=\frac{\left\langle\rho^{\prime}\left(z_{1}\right) \rho^{\prime}\left(z_{2}\right)\right\rangle}{\left\langle\rho^{\prime 2}\left(z_{1}\right)\right\rangle^{1 / 2}\left\langle\rho^{\prime 2}\left(z_{2}\right)\right\rangle^{1 / 2}},
$$

in figure $4(c, f)$, showing some non-trivial fringe-region effects for the shorter boxes, cases $\mathrm{A}$ and $\mathrm{B}$. As such, we recommend using tall boxes for simulating this flow. 

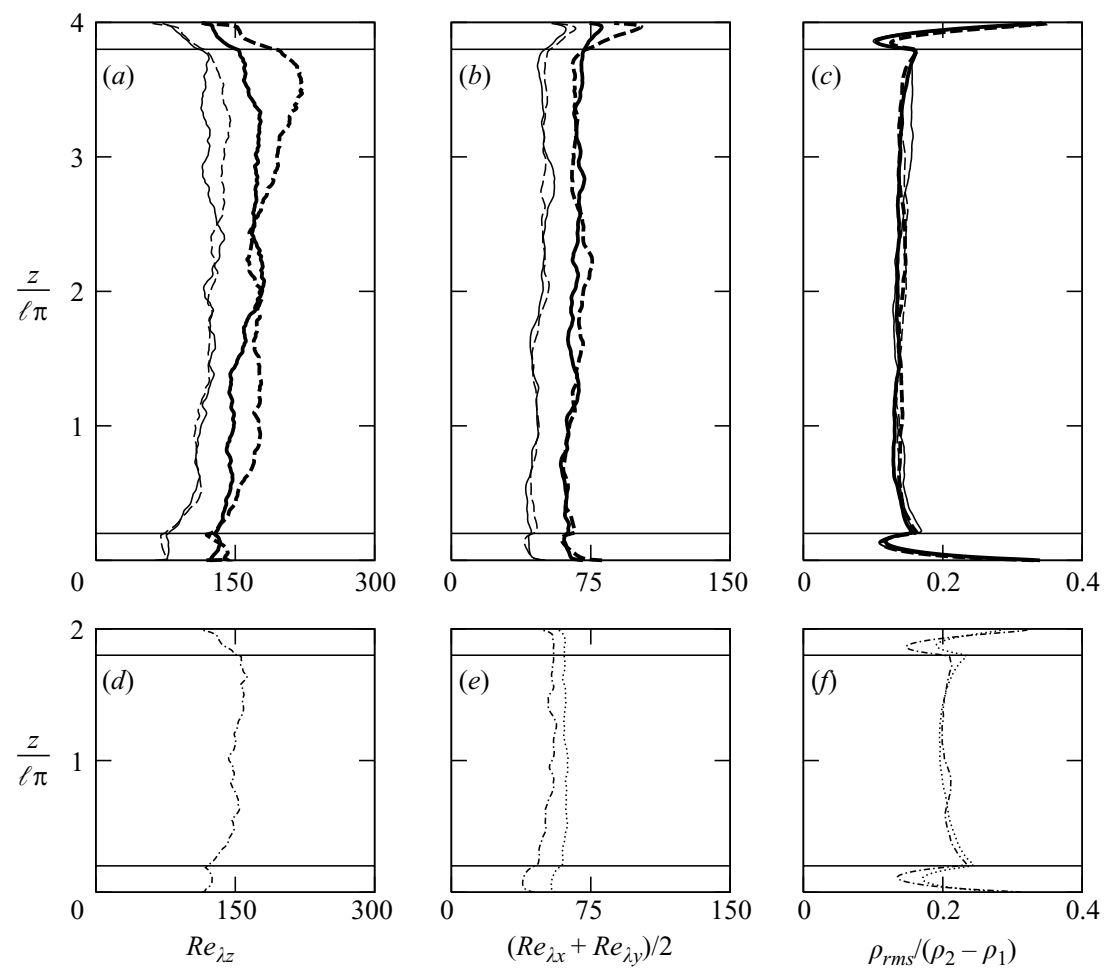

Figure 5. Profiles of Taylor-microscale Reynolds numbers, defined by (4.6), and density fluctuation intensities, defined by (4.7): $(a-c)$ tall boxes, $L_{z} / \ell=4 \pi ;(d-f)$ short boxes, $L_{z} / \ell=2 \pi$ (see table 1 for key). Horizontal lines indicate fringe-region boundaries. Case B $(\cdots \cdots \cdots)$, for which $R e_{\lambda_{z}} \approx 600$, is out of the range of $(d)$.

The Taylor-microscale Reynolds numbers are defined as

$$
\operatorname{Re}_{\lambda_{\alpha}}(z) \equiv \frac{\langle\rho\rangle \lambda_{\alpha}\left\langle u_{\alpha}^{\prime 2}\right\rangle^{1 / 2}}{\mu}, \quad \text { where } \quad \lambda_{\alpha}(z) \equiv\left(\frac{\left\langle u_{\alpha}^{\prime 2}\right\rangle}{\left\langle\left(\partial u_{\alpha}^{\prime} / \partial x_{\alpha}\right)^{2}\right\rangle}\right)^{1 / 2},
$$

no summation implied over $\alpha$. The profiles of the $R e_{\lambda_{z}}$ and $R e_{\lambda_{x y}} \equiv\left(R e_{\lambda_{x}}+R e_{\lambda_{y}}\right) / 2$, shown in figure 5 , indicate that, except for case $\mathrm{B}, R e_{\lambda_{z}} / R e_{\lambda_{x y}} \approx 2-2.5$. Such numbers are also reported by Cook \& Dimotakis (2001), with $R e_{\lambda_{z}} / R e_{\lambda_{x y}} \approx 2.5-4$, depending on the characteristic scale of their initial conditions. In case $\mathrm{B}$, however, $\operatorname{Re}_{\lambda_{z}} / \operatorname{Re}_{\lambda_{x y}} \approx 12$, perhaps owing to the large eddy sizes allowed by the horizontal extent of the computational domain. The general trend that $R e_{\lambda_{z}} / R e_{\lambda_{x y}}$ is higher with larger eddy sizes is also seen by Cook \& Dimotakis (2001).

The root-mean-square (r.m.s.) of density fluctuations at an $x y$-plane is given by

$$
\rho_{\text {rms }}(z)=\left\langle\rho^{\prime 2}\right\rangle^{1 / 2} \text {. }
$$

Its profiles for the tall boxes, but with different $R e$ and $A$, are plotted in figure $5(c)$. Outside the fringe, the profiles take on roughly constant values, scaling with $\left(\rho_{2}-\rho_{1}\right)$, regardless of $A$. In Cook et al. (2004), an effective Atwood number $A_{e}$, defined at the centre of the mixing zone as $\rho_{r m s} /\langle\rho\rangle$, is shown to approach $0.48 \mathrm{~A}$ at late times. Presently, $\langle\rho\rangle_{m} \approx \rho_{0}$ for all cases, and so $\left(A_{e} / A\right)=2 \rho_{r m s}\left(z=L_{z} / 2\right) /\left(\rho_{2}-\rho_{1}\right)=0.35-0.4$, depending on the aspect ratio of the computational domain. 

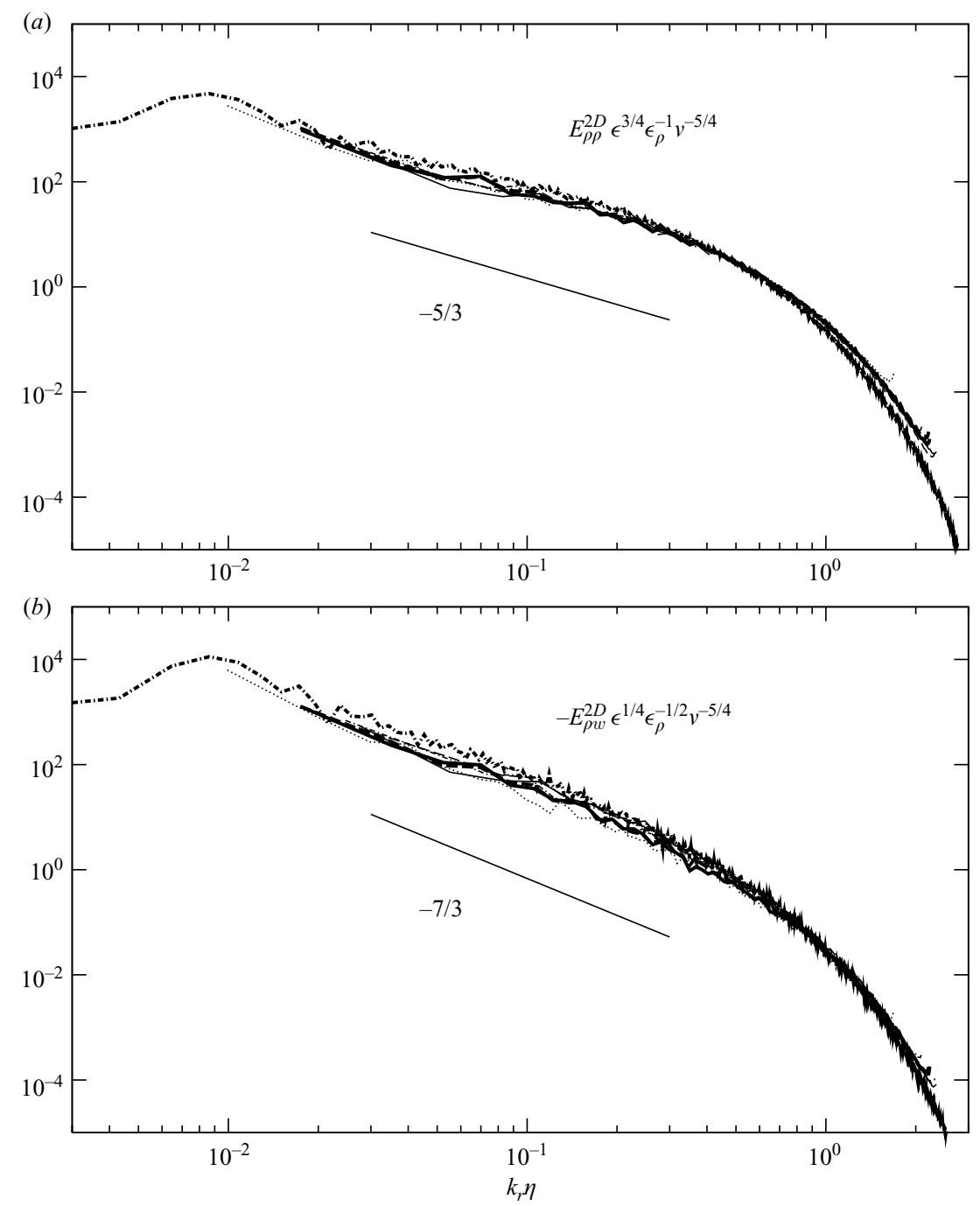

FIGURE 6. Mid-plane spectra normalized by KOC scales (4.8) of $(a)$ density and (b) density-vertical-velocity: -.-.--, $3072^{3}$ DNS of Rayleigh-Taylor instability (Cabot \& Cook 2006); remaining lines are from present simulations (see table 1 for key).

\subsection{Spectra}

The planar spectra, $E_{\rho \rho}^{2 D},-E_{\rho w}^{2 D}, E_{u u}^{2 D}$ and $E_{v v}^{2 D}$, as defined by (4.4), at the mid-plane location, are plotted in figures 6 and 7, non-dimensionalized by their respective midplane Kolmogorov-Obukhov-Corrsin (KOC) scales: specific kinetic energy dissipation $\epsilon$, density fluctuation dissipation $\epsilon_{\rho}$ and kinematic viscosity $\nu$, which we will define as

$$
\epsilon=v\left\langle\left(\frac{\partial u_{i}}{\partial x_{j}}\right)^{2}+\frac{1}{3}\left(\frac{\partial u_{i}}{\partial x_{i}}\right)^{2}\right\rangle_{m}, \quad \epsilon_{\rho}=\mathscr{D}\left\langle\left(\frac{\partial \rho}{\partial x_{j}}\right)^{2}\right\rangle_{m}, \quad v=\frac{\mu}{\langle\rho\rangle_{m}},
$$

from which $\eta=\left(v^{3} / \epsilon\right)^{1 / 4}$. The present simulations are well resolved $-k_{\max } \eta \approx 2$ in all cases save case $\mathrm{B}$, for which is it $k_{\max } \eta \approx 1.6$. Observe that, when plotted in Kolmogorov scaling, all the spectra from the present simulations virtually collapse, 

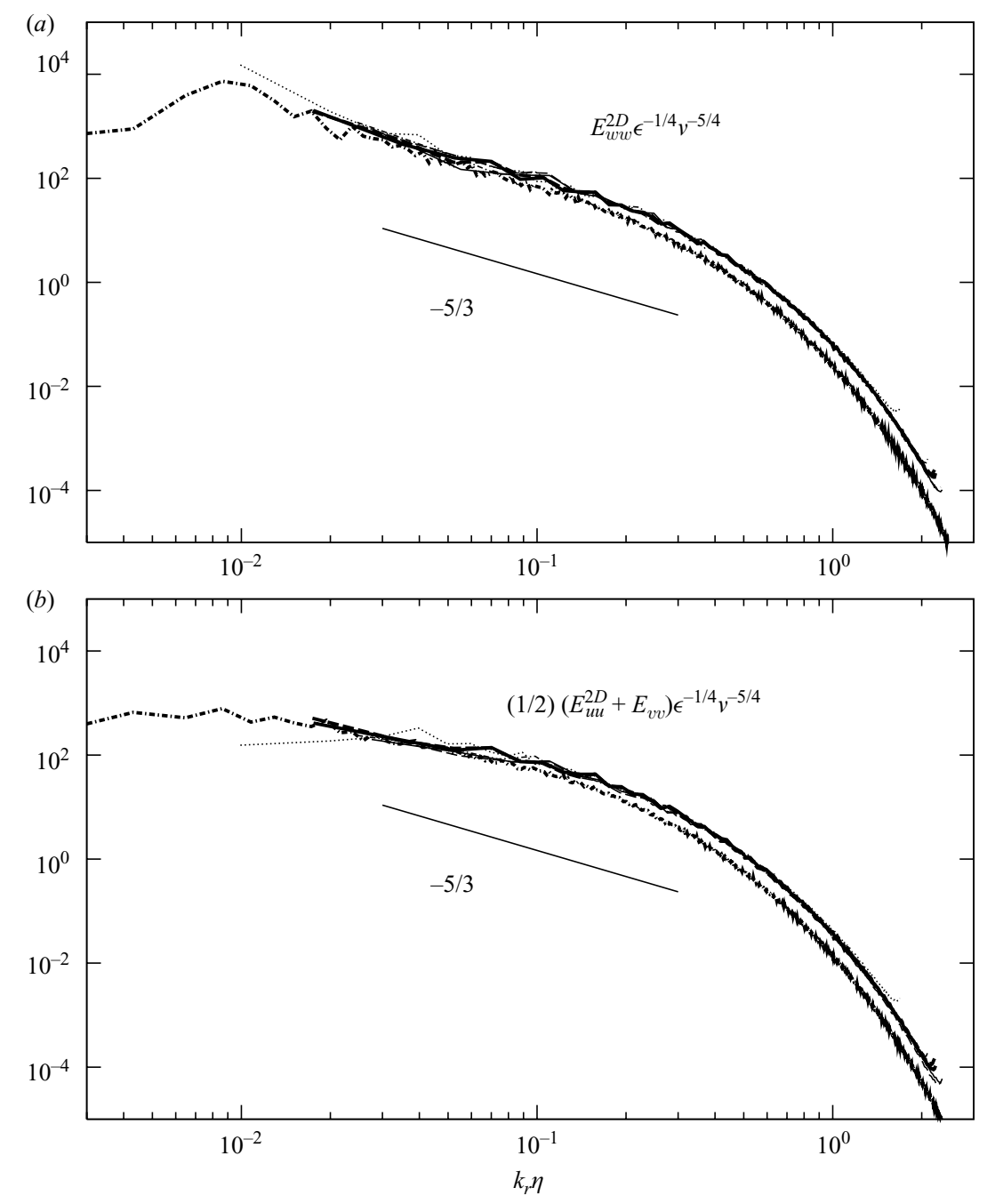

FIGURE 7. Mid-plane spectra normalized by KOC scales (4.8) of $(a)$ vertical velocity and (b) horizontal velocity: ---.---, $3072^{3}$ DNS of Rayleigh-Taylor instability (Cabot \& Cook 2006); remaining lines are from present simulations (see table 1 for key).

especially in the high-wavenumber range, regardless of $A, R e$ and $L_{z} / L_{x}$. This suggests that, in modelling spectra, the standard scaling ideas (Lumley 1967) used for passive scalar mixing can still be applied to the active scalar mixing problem; in other words, $\epsilon, \epsilon_{\rho}$ and $\nu$ are still the relevant inner scales. Furthermore, these spectra appear to approach the standard power-law scaling with the $-5 / 3$ exponent for $E_{w w}^{2 D}$ and $\left(E_{u u}^{2 D}+E_{v v}^{2 D}\right) / 2$, and the $-7 / 3$ exponent for $-E_{\rho w}^{2 D}$.

For comparison, we also show the $3072^{3}$ DNS spectra from Cabot \& Cook (2006) in figures 6 and 7, normalized by their constant- $\nu$ version of (4.8). We also ran a constant $-v$, that is $\mu(\boldsymbol{x}, t)=v \rho(\boldsymbol{x}, t)$, version of the present flow simulations with no discernible differences in the spectra. The present data allow the comparison of statistically evolving Rayleigh-Taylor spectra relative to statistically stationary flow spectra at the same level of dissipation (same inner scaling). Specifically, when compared with Rayleigh-Taylor spectra, $E_{\rho \rho}^{2 D}$ and $-E_{\rho w}^{2 D}$ show near collapse but the 
present $E_{w w}^{2 D}$ and $\left(E_{u u}^{2 D}+E_{v v}^{2 D}\right) / 2$ are higher in the high-wavenumber range. This is perhaps not surprising since turbulence production is greater than dissipation in Rayleigh-Taylor turbulence, whereas the present spectra represent 'equilibrium' buoyancy-driven turbulence. Even though they do not collapse completely, they share common power-law slopes in the incipient inertial range.

The present density spectra exhibit slopes somewhat flatter than $-5 / 3$ (figure $6 a$ ), a phenomenon reported by Warhaft (2000) for passive scalar spectra and also apparent in the Cabot \& Cook (2006) spectrum. For the passive-scalar case, figure 4 of Warhaft (2000) suggests that this could be a low-Re effect (see also Miller 1991). Consistent with this trend, the higher-Re Rayleigh-Taylor density spectrum (figure $6 a$ ) exhibits a slope closer to $-5 / 3$. The stretched-spiral vortex model for turbulent scalar mixing (Pullin \& Lundgren 2001), formally an asymptotic large-time Navier-Stokes solution, provides a theoretical framework to explain this phenomenon. According to the model, the density spectrum comprises additive contributions from mixing by nonaxisymmetric vorticity and axisymmetric components of the velocity field giving rise to $k^{-5 / 3}$ and $k^{-1}$ spectra, respectively (Pullin \& Lundgren 2001, equations (107) and (108)). The latter, which is subdominant at large $R e$, has the effect of flattening the composite scalar spectrum.

\subsection{Mole fraction probability density functions}

The probability density functions (p.d.f.s) of $X_{2}$, shown in figure 8 , are taken at various vertical locations: the leftmost and rightmost curves represent the p.d.f.s taken from the middle of the lower and upper fringes, respectively, and the remaining curves are p.d.f.s taken from the quarter-, half- and three-quarter domain height. Outside the fringe regions, the p.d.f.s are roughly unimodal with peaks varying from 0 to 1 . An exception is case B (figure $8 b$ ), which exhibits bimodal behaviour, indicating the persistence of unmixed fluids. This can be attributed to the large eddies, permitted by the large horizontal dimensions (figure $4 d$ ), that cause large-scale sloshing motions as unmixed fluids clump together. In contrast, we observe better small-scale mixing when the eddies are smaller (cases $\mathrm{A}, \mathrm{C}-\mathrm{F}$ ). All else equal, the $A=3 / 4$ runs (figure $8 d, f$ ) exhibit wider p.d.f.s compared with the $A=1 / 2$ runs (figure $8 c, e$ ). We also observe a slight skew towards lower $X_{2}$ at the mid-plane, seen in figure $8(c-f)$, consistent with the Rayleigh-Taylor turbulence simulation performed by (Cook et al. (2004; figure 13).

\section{An a posteriori test of a subgrid-scale scalar flux model}

The present DNS data provide an opportunity to test subgrid-scale (SGS) modelling ideas for LES in the context of statistically stationary unstably stratified turbulent flows. Considered as part of a multi-scale framework, each of the present simulations can be viewed as a unit of statistically stationary turbulent flow found in an LES cell that is embedded in a density gradient. The LES-SGS modelling exercise poses the following question as a means for turbulence closure: given resolved-scale quantities, for example velocity gradients and scalar gradients, what are the SGS stresses and SGS scalar fluxes? Presently, we will focus on the SGS scalar flux.

By considering the action of an ensemble of axisymmetric subgrid vortices on a passive scalar $\psi(\boldsymbol{x})$, Pullin (2000) developed a model for the SGS scalar flux, which was also subsequently used by Hill, Pantano \& Pullin (2006) to model SGS heat flux in the Richtmyer-Meshkov instability. Assuming that the ensemble of subgrid vortices is orthogonal to the vertical, which is the case for baroclinic vorticity that 

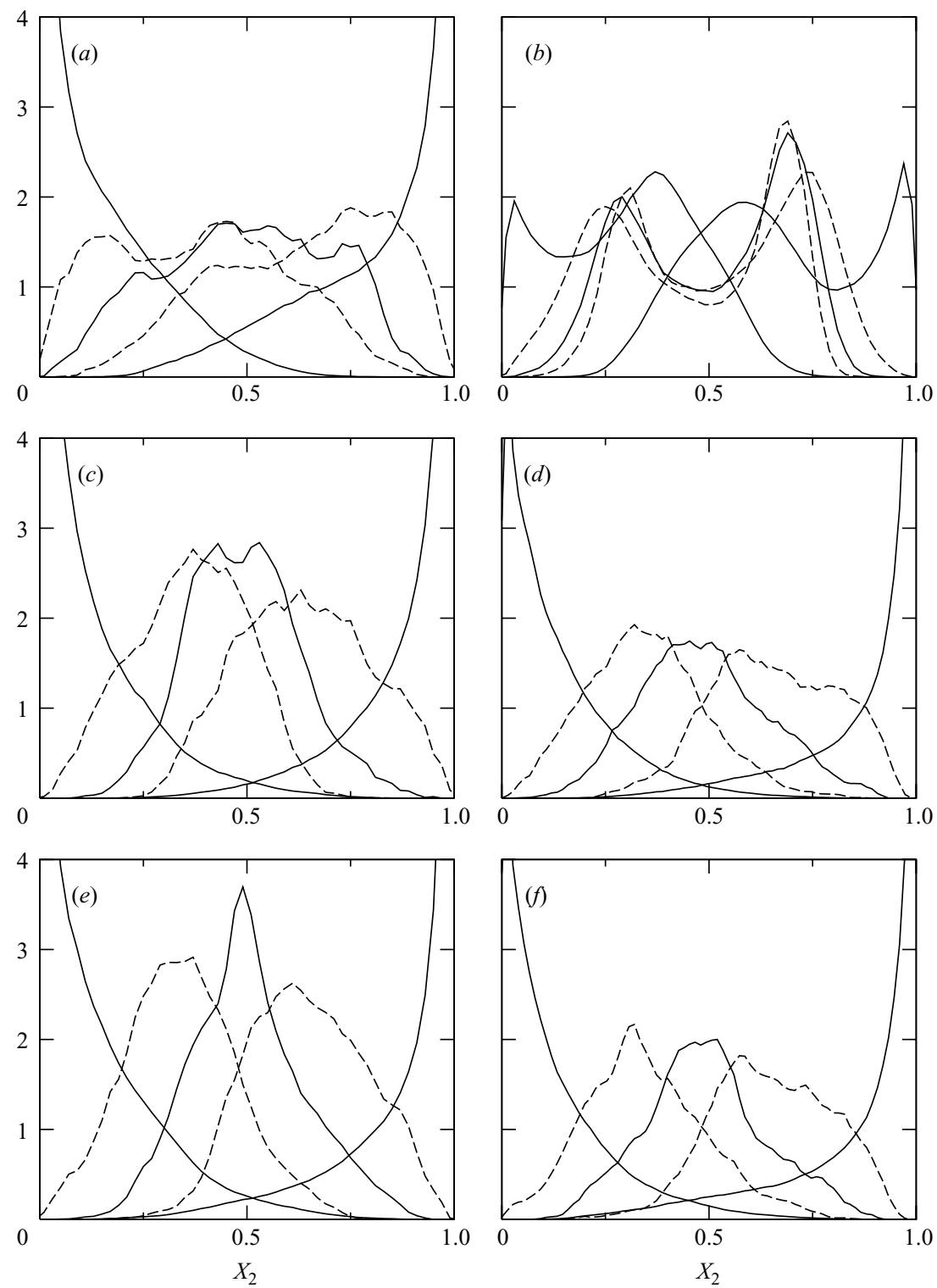

FIGURE 8. P.d.f.s of $X_{2}$, defined by (4.2), taken from $x y$-planes located at, from left to right (alternating between solid and broken lines for legibility), $z=0.5 L_{f}, 0.25 L_{z}, 0.5 L_{z}, 0.75 L_{z}$ and $L_{z}-0.5 L_{f} .(a)$ Case A, $(b)$ case $\mathrm{B},(c)$ case $\mathrm{C},(d)$ case $\mathrm{D},(e)$ case $\mathrm{E}$ and $(f)$ case $\mathrm{F}$.

accompanies bubble and spikes in unstably stratified flows, the general expression for the modelled (specific) SGS passive scalar flux $q_{z}^{\psi}$ takes the following form (see Hill et al. 2006):

$$
q_{z}^{\psi}=-\gamma_{\psi} \frac{\pi}{2 k_{c}} K^{1 / 2} \frac{\partial \widetilde{\psi}}{\partial z}, \quad \text { where } \quad q_{z}^{\psi} \equiv \widetilde{\psi w}-\widetilde{\psi} \widetilde{w}
$$

where the LES Favre average for any field $f$ is given by $\widetilde{f} \equiv \overline{\rho f} / \bar{\rho}$, which, in turn, is defined in terms of the LES (low-pass) filter $\bar{f}$ associated with the cutoff wavenumber $k_{c} ; K=\left(\overline{u_{i} u_{i}}-\overline{\overline{u_{i}}} \overline{\overline{u_{i}}}\right) / 2$, the (specific) SGS kinetic energy; and $\gamma_{\psi}$ is 


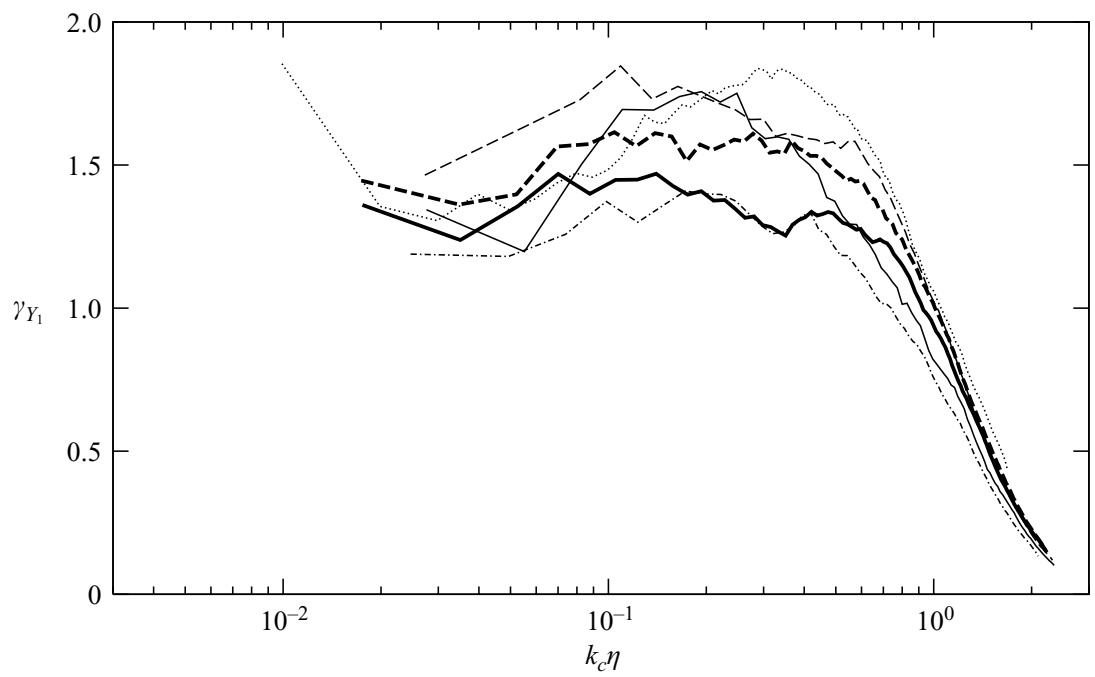

FIGURE 9. The mixing-time model constant $\gamma_{Y_{1}}\left(k_{c}\right)$ of the vortex-based SGS scalar flux model of Pullin (2000) computed from the present DNS data as an a posteriori test. See table 1 for key.

the $O(1)$ mixing-time constant presumed to be universal. Pullin (2000) estimated that $\gamma_{\psi}=0.89-1.3$.

We now consider a model for an active scalar, the mass fraction $Y_{1}$, using an analogous framework: put $\psi=Y_{1}$ in (5.1), then write the resulting expression in terms of $\rho$ via the algebraic relation (2.5) to obtain

$$
\overline{\rho w}-\bar{\rho} \bar{w}=-\gamma_{Y_{1}} \frac{\pi}{2 k_{c}} K^{1 / 2} \frac{\partial \bar{\rho}}{\partial z} .
$$

Interpreting the homogeneous directions of the DNS domain as a model LES cell, we identify the LES average $\overline{()}$ with the DNS plane average \langle\rangle and the LES fluctuations $f-\bar{f}$ with the DNS plane fluctuations $f^{\prime}$. Substituting these into (5.2), we obtain

$$
\gamma_{Y_{1}}=\frac{-\left\langle\rho^{\prime} w^{\prime}\right\rangle}{\left(\pi /\left(2 k_{c}\right)\right)\left(\left\langle u_{i}^{\prime} u_{i}^{\prime}\right\rangle / 2\right)^{1 / 2}(\partial\langle\rho\rangle / \partial z)} .
$$

The a posteriori test of the SGS scalar flux model then consists of verifying that the mixing-time constant is $O(1)$. To properly assess the validity of (5.3), we eliminate the effects of the computational box size, which may influence the low wavenumbers, by replacing all plane averages $\left\langle f^{\prime} g^{\prime}\right\rangle$ in (5.3) with the scale-dependent

$$
\left\langle f^{\prime} g^{\prime}\right\rangle\left(k_{\mathrm{c}}\right)=\int_{k_{\mathrm{c}}}^{\infty} E_{f g}^{2 D}\left(k_{r}\right) \mathrm{d} k_{r} .
$$

Plotting $\gamma_{Y_{1}}\left(k_{\mathrm{c}}\right)$ for all the present DNS in figure 9, we observe that, in agreement with the analysis by Pullin (2000), it is indeed an $O(1)$ quantity taking on roughly constant values in the inertial range with some scatter 1.2-1.8 over one decade of wavenumbers before dropping to zero near $k_{c} \eta=1$. This observation is rather surprising, since we have applied a passive scalar model $(R=1)$ to an active scalar with moderately high $R=3,7$. A possible explanation is that, regardless of $R$, the present simulations of unstably stratified flows are still rate-limited by turbulent mixing, which is a key argument used in the development of the passive scalar flux 
model. One could imagine a different outcome in a stably stratified configuration, where stable stratification overwhelms turbulent mixing by converting kinetic energy related to counter-gradient motion into potential energy, effectively damping the flow (high Richardson numbers).

\section{Large-eddy simulation}

In addition to serving as a posteriori test beds for SGS models, the present DNSs are excellent test cases for LES - the turbulence is statistically stationary, anisotropic and buoyancy-driven. In what follows, we describe LES of these DNSs, focusing on LES-specific details. Following Hill et al. (2006), we filter the governing equations (2.1)-(2.3) and (2.5), and then rearrange them to obtain:

$$
\begin{gathered}
\frac{\partial \widetilde{u}_{j}}{\partial x_{j}}=-\mathscr{D} \frac{\partial^{2} s}{\partial x_{j}^{2}}+\frac{\partial q_{j}^{s}}{\partial x_{j}}-\omega_{s}, \\
\frac{\partial s}{\partial t}+\widetilde{u}_{j} \frac{\partial s}{\partial x_{j}}=\mathscr{D} \frac{\partial^{2} s}{\partial x_{j}^{2}}-\frac{\partial q_{j}^{s}}{\partial x_{j}}+\omega_{s}, \\
\frac{\partial \widetilde{u}_{i}}{\partial t}+\widetilde{u}_{j} \frac{\partial \widetilde{u}_{i}}{\partial x_{j}}=-\frac{1}{\bar{\rho}}\left(\frac{\partial \bar{p}}{\partial x_{i}}+\Gamma_{i}\right)+\frac{1}{\bar{\rho}} \frac{\partial \widetilde{\tau}_{i j}}{\partial x_{j}}-\frac{1}{\bar{\rho}} \frac{\partial \bar{\rho} T_{i j}}{\partial x_{j}}-g \delta_{i 3}, \\
1 / \bar{\rho}=\widetilde{Y}_{1} / \rho_{1}+\left(1-\widetilde{Y}_{1}\right) / \rho_{2},
\end{gathered}
$$

where $s \equiv \log \left(\bar{\rho} / \rho_{0}\right)$, and $\widetilde{()}$ and $\overline{()}$ are, respectively, the Favre and LES averages described in $\S 5$. We use the stretched-spiral vortex SGS model (Misra \& Pullin 1997; Voelkl, Pullin \& Chan 2000), in which the SGS motion is represented by an ensemble of stretched vortices (Lundgren 1982) that are local two-dimensional large-time Navier-Stokes solutions. The SGS model stress tensor is given by

$$
T_{i j} \equiv \widetilde{u_{i} u_{j}}-\widetilde{u_{i}} \widetilde{u_{j}}=\left(\delta_{i j}-e_{i}^{v} e_{j}^{v}\right) K,
$$

where we have assumed that the subgrid vortices are aligned according to deltafunction orientation p.d.f.s with peaks at $e_{i}^{v}$. Presently, we choose the unit vector $e_{i}^{v}$ to coincide with the most extensive eigenvector of the strain-rate tensor $\widetilde{S_{i j}}=\left(\partial \widetilde{u_{i}} / \partial x_{j}+\right.$ $\left.\partial \widetilde{u_{j}} / \partial x_{i}\right) / 2$. The subgrid (specific) kinetic energy is given by

$$
K=\int_{k_{c}}^{\infty} E(\kappa) \mathrm{d} \kappa
$$

where the cutoff wavenumber, $k_{c} \equiv \pi / \Delta_{c}$, and presently $\Delta_{c}=\Delta_{x}=\Delta_{y}=\Delta_{z}$. The energy spectrum of a stretched-spiral vortex is (Lundgren 1982)

$$
E(\kappa)=A \kappa^{-5 / 3} \exp \left(-\kappa^{2} \lambda_{v}^{2}\right),
$$

where $\lambda_{v}=(2 \widetilde{v} /(3|\widetilde{a}|))^{1 / 2} ; \widetilde{v}=\mu / \bar{\rho} ;$ and $\widetilde{a}=e_{i}^{v} e_{j}^{v} \widetilde{S_{i j}}$, which represents the axial stretching of subgrid vortices by resolved scales. Combining (6.3) and (6.4), we obtain

$$
K=A \lambda_{v}^{2 / 3} \frac{1}{2} \Gamma_{-1 / 3}\left(k_{c}^{2} \lambda_{v}^{2}\right),
$$

where $\Gamma_{-1 / 3}($ ) is an incomplete Gamma function. We determine the inseparable group prefactor $A=\mathscr{K}_{0} \epsilon^{2 / 3}$ by matching an expression for the SGS model structure function with its observed value computed from the resolved part of the LES simulation (Pullin 2000; Voelkl et al. 2000; Hill et al. 2006). 


\begin{tabular}{cccccccccccccc}
\hline \multirow{2}{*}{ Key } & Case & $R e$ & $S c$ & $A$ & $R$ & $L_{x} / \ell$ & $L_{y} / \ell$ & $L_{z} / \ell$ & $L_{f} / \ell$ & $N_{x}$ & $N_{y}$ & $N_{z}$ & $T_{e}$ \\
$\circ$ & $\mathrm{E} 1$ & 478 & 1 & $1 / 2$ & 3 & $2 \pi$ & $2 \pi$ & $4 \pi$ & $2 \pi / 10$ & 32 & 32 & 64 & 23.1 \\
$\square$ & $\mathrm{E} 2$ & 478 & 1 & $1 / 2$ & 3 & $2 \pi$ & $2 \pi$ & $4 \pi$ & $2 \pi / 10$ & 64 & 64 & 128 & 22.5 \\
$\diamond$ & $\mathrm{E} 3$ & 478 & 1 & $1 / 2$ & 3 & $2 \pi$ & $2 \pi$ & $4 \pi$ & $2 \pi / 10$ & 128 & 128 & 256 & 9.1 \\
& $\mathrm{E}$ & 478 & 1 & $1 / 2$ & 3 & $2 \pi$ & $2 \pi$ & $4 \pi$ & $2 \pi / 10$ & 384 & 384 & 768 & 5.3
\end{tabular}

TABLE 2. LES parameters. $T_{e}$ is defined in (4.1). Cases E1, E2 and E3 are LES and case E is DNS (also in table 1).

We model the (specific) flux of $Y_{1}$ as if it were a passive scalar (Pullin 2000; Hill et al. 2006; see also §5):

$$
q_{i}^{Y_{1}} \equiv \widetilde{Y_{1} u_{i}}-\widetilde{Y}_{1}{\widetilde{u_{i}}}=-\gamma_{Y_{1}} \frac{\pi}{2 k_{c}} K^{1 / 2}\left(\delta_{i j}-e_{i}^{v} e_{j}^{v}\right) \frac{\partial \widetilde{Y}_{1}}{\partial x_{j}} ;
$$

presently, $\gamma_{Y_{1}}=1$. Upon substituting (6.1d) into (6.6) and comparing the result with both the filtered form of $(2.1)$ and $(6.1 b)$, we find that

$$
q_{i}^{s}=\tilde{u_{i}}-\overline{u_{i}}=-\left(\frac{1}{\rho_{1}}-\frac{1}{\rho_{2}}\right) \bar{\rho} q_{i}^{Y_{1}}=-\gamma_{Y_{1}} \frac{\pi}{2 k_{c}} K^{1 / 2}\left(\delta_{i j}-e_{i}^{v} e_{j}^{v}\right) \frac{\partial s}{\partial x_{j}} .
$$

We rearrange this to relate the SGS mass flux, to the resolved density gradient:

$$
\overline{\rho u_{i}}-\bar{\rho} \overline{u_{i}}=-\gamma_{Y_{1}} \frac{\pi}{2 k_{c}} K^{1 / 2}\left(\delta_{i j}-e_{i}^{v} e_{j}^{v}\right) \frac{\partial \bar{\rho}}{\partial x_{j}} .
$$

We now show that this SGS scalar flux model contains an intrinsic power-law inertialrange scaling for the scalar-velocity cospectrum with a $-7 / 3$ exponent. By definition, (6.7) is also equal to $\int_{k_{c}}^{\infty} E_{\rho u_{i}}\left(\kappa ; \boldsymbol{e}^{v}\right) \mathrm{d} \kappa$, where $E_{\rho u_{i}}$ is the $\rho-u_{i}$ cospectrum of the two-dimensional flow in the $\boldsymbol{e}^{v}$-oriented vortex cross-section. Substituting (6.5) into (6.7), we solve for $E_{\rho u_{i}}$ to get

$$
E_{\rho u_{i}}\left(\kappa ; \boldsymbol{e}^{v}\right)=-\gamma_{Y_{1}}(2 / 3)^{1 / 2} \pi A^{1 / 2} \lambda_{v}^{7 / 3} F\left(\kappa \lambda_{v}\right)\left(\delta_{i j}-e_{i}^{v} e_{j}^{v}\right) \frac{\partial \bar{\rho}}{\partial x_{j}},
$$

where

$$
F\left(\kappa_{v}\right)=\frac{\sqrt{3}}{4} \kappa_{v}^{-7 / 3} \frac{\exp \left(-\kappa_{v}^{2}\right)+\kappa_{v}^{2 / 3} \Gamma_{-1 / 3}\left(\kappa_{v}^{2}\right)}{\left(\kappa_{v}^{2 / 3} \Gamma_{-1 / 3}\left(\kappa_{v}^{2}\right)\right)^{1 / 2}} .
$$

In the inertial range $\left(\kappa \lambda_{v} \ll 1\right), F \sim \kappa^{-7 / 3}$, so (6.8) is consistent with the well-known result obtained from scaling arguments (Lumley 1967) that $E_{\rho u}(\kappa) \propto(\partial \bar{\rho} / \partial x) \epsilon^{1 / 3} \kappa^{-7 / 3}$. We perform LES at three different resolutions of the present DNS case E (see table 2).

\subsection{Subgrid extensions of planar spectra}

Assuming that the subgrid vortices are aligned according to delta-function p.d.f.s with peaks at

$$
\boldsymbol{e}^{v}(\boldsymbol{x})=\left(\sin \alpha_{0} \cos \beta_{0}, \sin \alpha_{0} \sin \beta_{0}, \cos \alpha_{0}\right),
$$

we use the following expressions, given by Hill et al. (2006), (6.11) and (6.12) in that paper, to obtain $x y$-plane velocity spectra from spiral-vortex spectra (see also Pullin 
\& Saffman 1994):

$$
\begin{aligned}
& E_{q q}^{2 D}\left(k_{r}\right)=\frac{2 k_{r}}{\pi} \int_{k_{r}}^{\left|k_{r} / \cos \alpha_{0}\right|} \frac{E(\kappa)}{\left(\kappa^{2}-k_{r}^{2}\right)^{1 / 2}\left(k_{r}^{2}-\kappa^{2} \cos ^{2} \alpha_{0}\right)^{1 / 2}} \mathrm{~d} \kappa, \\
& E_{33}^{2 D}\left(k_{r}\right)=\frac{2 k_{r}}{\pi} \int_{k_{r}}^{\left|k_{r} / \cos \alpha_{0}\right|} \frac{\left(k_{r}^{2}-\kappa^{2} \cos ^{2} \alpha_{0}\right)^{1 / 2} E(\kappa)}{\kappa^{2}\left(\kappa^{2}-k_{r}^{2}\right)^{1 / 2}} \mathrm{~d} \kappa,
\end{aligned}
$$

with $E(\kappa)$ is given by (6.4). In the present notation, $\left(E_{u u}^{2 D}+E_{v v}^{2 D}\right) / 2=E_{q q}^{2 D}-E_{33}^{2 D}$ and $E_{w w}^{2 D}=2 E_{33}^{2 D}$. A similar expression can be found for the planar $\rho-u_{i}$ cospectrum:

$$
E_{\rho u_{i}}^{2 D}\left(k_{r}\right)=\frac{2 k_{r}}{\pi} \int_{k_{r}}^{\left|k_{r} / \cos \alpha_{0}\right|} \frac{E_{\rho u_{i}}\left(\kappa ; \boldsymbol{e}^{v}\right)}{\left(\kappa^{2}-k_{r}^{2}\right)^{1 / 2}\left(k_{r}^{2}-\kappa^{2} \cos ^{2} \alpha_{0}\right)^{1 / 2}} \mathrm{~d} \kappa,
$$

where $E_{\rho u_{i}}\left(\kappa ; \boldsymbol{e}^{v}\right)$ is given by (6.8). Given $k_{r}$ and $z$, we average (6.9) and (6.10) across the $x y$-plane to obtain subgrid extensions of planar spectra.

We plot in figure 10 mid-plane resolved-scale spectra and their subgrid extensions, normalized by $v \equiv \mu /\langle\bar{\rho}\rangle$ and $\epsilon^{\prime} \equiv\left\langle\widetilde{S_{i j}} \widetilde{\tau_{i j}}-\bar{\rho} \widetilde{S_{i j}} T_{i j}\right\rangle /\langle\bar{\rho}\rangle$. The LES spectra are in general agreement with their DNS counterparts, also shown in figure 10. However, the subgrid extensions show noticeable resolution dependence at the viscous roll-off. This can be understood as follows: the viscous roll-off is determined by the factor $\exp \left(-2 \widetilde{v} \kappa^{2} /(3|\widetilde{a}|)\right)$ in the model energy spectrum, (6.4), but the local strain rate $\widetilde{a}$, scaling like $\int_{0}^{k_{c}} k^{2} E(k) \mathrm{d} k$, is itself an LES resolution-dependent quantity. Since we expect that the energy transfer off the resolved-scale grid to subgrid scales will, in general, depend on the LES resolution, then this approximation is acceptable and necessary for integrating the resolved-scale variables in time. For the purposes of subgrid extension, however, a different approximation is appropriate.

Following Lundgren (1982), we use the estimate from isotropic turbulence that $\widetilde{a}=(\widetilde{\epsilon} /(15 \widetilde{v}))^{1 / 2}$, where $\widetilde{\epsilon}$ is the local cell-averaged dissipation rate to be determined. With this estimate, $\lambda_{v} \approx 1.61 \widetilde{\eta}$ where $\widetilde{\eta}=\left(\widetilde{v}^{3} / \widetilde{\epsilon}\right)^{1 / 4}$, so that the viscous roll-off is now characterized by the resolution-independent factor $\exp \left(-(1.61 \widetilde{\eta})^{2} \kappa^{2}\right)$ in $(6.4)$. To determine $\widetilde{\epsilon}$, we solve the following transcendental equation, obtained from (6.2), (6.5) and the definitions of $\widetilde{\epsilon}$ and $\widetilde{\eta}$ :

$$
\left.\begin{array}{l}
\widetilde{\epsilon}=\widetilde{S}_{i j} \tau_{i j} / \bar{\rho}-\widetilde{S}_{i j}\left(\delta_{i j}-e_{i}^{v} e_{j}^{v}\right) \frac{1}{2} A(1.61 \widetilde{\eta})^{2 / 3} \Gamma\left[-1 / 3,(1.61 \widetilde{\eta})^{2} k_{c}^{2}\right], \\
\widetilde{\eta}=\left(\widetilde{v}^{3} / \widetilde{\epsilon}\right)^{1 / 4}
\end{array}\right\}
$$

where $A$ is the inseparable group prefactor $\mathscr{K}_{0} \epsilon^{2 / 3}$ determined from the structure function matching procedure. Equation (6.11), analogous to (37) of Pullin \& Saffman (1993), merely states that the local cell-averaged dissipation comprises the resolved and subgrid components. Having determined $\widetilde{\epsilon}$, we can then calculate the subgrid extensions as before but replacing $\lambda_{v}=1.61 \tilde{\eta}$ in both (6.4) and (6.8).

We re-plot in figure $11(a)$ the mid-plane resolved-scale velocity spectra and their subgrid extensions, now normalized by $v$ and $\epsilon$, where $v \equiv \mu /\langle\bar{\rho}\rangle$ as before, but now $\epsilon=\langle\widetilde{\epsilon}\rangle$, different from $\epsilon^{\prime}$. The general features of the LES spectra are in agreement with their DNS counterparts. However, LES slightly underpredicts both the verticalvelocity spectrum, $E_{w w}^{2 D}$, and the horizontal-velocity spectrum, $\left(E_{u u}^{2 D}+E_{v v}^{2 D}\right) / 2$, at around $k_{r} \eta=10^{-1}$. Observe that the improved subgrid extensions now collapse. The $\rho-w$ cospectra, normalized by $\nu, \epsilon$ and $\langle\partial \rho / \partial z\rangle$, are shown in figure $11(b)$. The resolved part of the LES cospectra agrees well with DNS and their subgrid extensions 

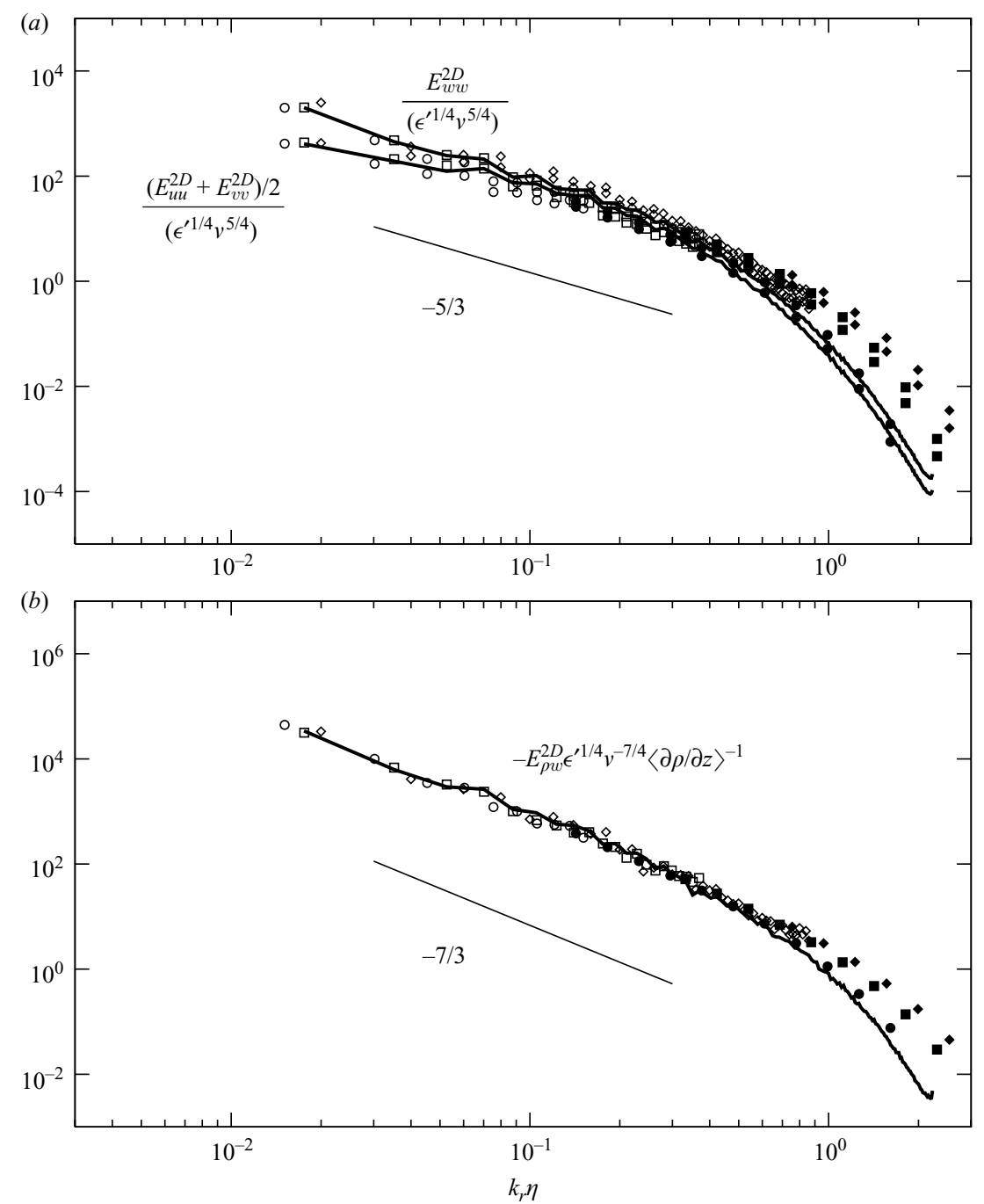

FIGURE 10. LES and DNS comparisons, with $\lambda_{v}=\left(2 \widetilde{v} /(3|\widetilde{a}|)^{1 / 2}\right.$, of $(a)$ mid-plane vertical-velocity spectra and horizontal-velocity spectra and $(b)$ mid-plane density-vertical-velocity cospectra: open symbols, resolved; solid symbols, subgrid; see table 2 for key.

accurately capture the shape and location of the viscous roll-off, independent of the LES resolution.

We remark that in the subgrid estimation, $\widetilde{\epsilon}$ is to be viewed as a random variable whose planar and temporal p.d.f. is determined by the LES itself. Pullin \& Saffman (1993) argue that the effect of statistical variation of the effective strain can change the $-\kappa^{2}$ factor within the exponential of the Lundgren spectrum to $-\kappa$, as observed in the experiment and DNS. This effect can be seen in the log-linear plot of the present subgrid-scale extensions (figure 12) that show a trend close to $\exp \left(-\beta k_{r} \eta\right)$ where $\beta \approx 5.2$, a result consistent with Saddoughi \& Veeravalli (1994), $\beta=5.2$; Martínez et al. (1997), $\beta=4.5-6$; and Schumacher (2007), $\beta=3-8$. We quote ranges for $\beta$ because these studies show that $\beta$ is sensitive to $R e_{\lambda}$. Furthermore, the value of $\beta$ 

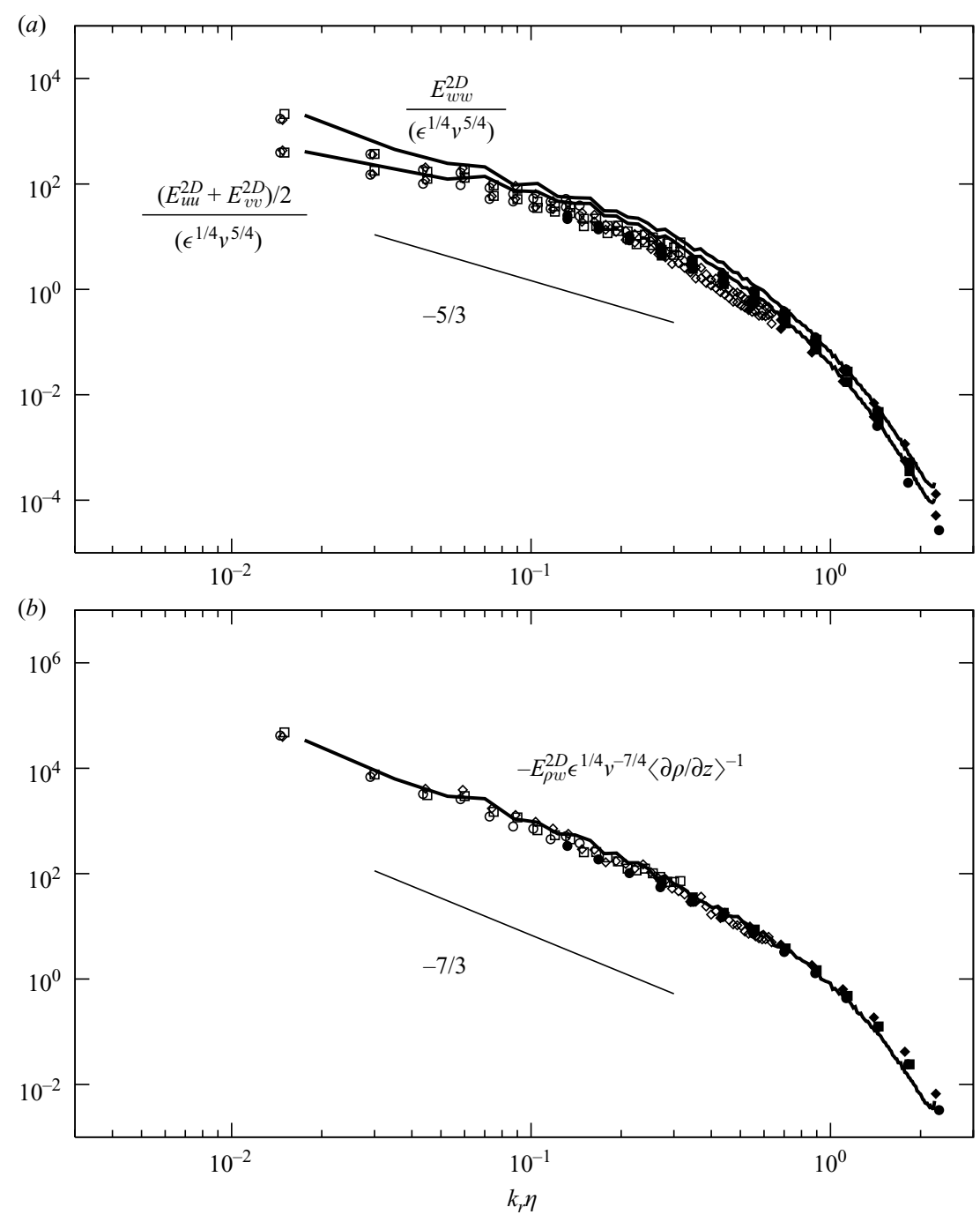

FIGURE 11. LES and DNS comparisons, with $\lambda_{v}=1.61 \widetilde{\eta}$, of $(a)$ mid-plane vertical-velocity spectra and horizontal-velocity spectra and $(b)$ mid-plane density-vertical-velocity cospectra: open symbols, resolved; solid symbols, subgrid; see table 2 for key.

from the present two-dimensional spectra, and also the one-dimensional spectra of Saddoughi \& Veeravalli (1994), could be obscured by aliasing.

Plotting the velocity-anisotropy parameter $E_{w w}^{2 D} / E_{u_{i} u_{i}}^{2 D}-1 / 3$ in figure $13(a)$, we observe that the present LES captures the small-scale anisotropy observed in DNS. In particular, the decreasing anisotropy from low wavenumbers up to $k_{r} \eta=10^{-1}$, followed by a gradually increasing anisotropy up to $k_{r} \eta=2$, is reported by both DNS and LES. For the LES, the location of the minimum is independent of the LES resolution. It appears from figure 13(a) that the anisotropy increases indefinitely, albeit gradually, with increasing wavenumber. We superimpose the high-resolution Rayleigh-Taylor DNS velocity-anisotropy parameter from Cabot \& Cook (2006) in figure $13(b)$ to see that the anisotropy eventually decreases after reaching a local maximum of about 0.18 at $k_{r} \eta=2$. 


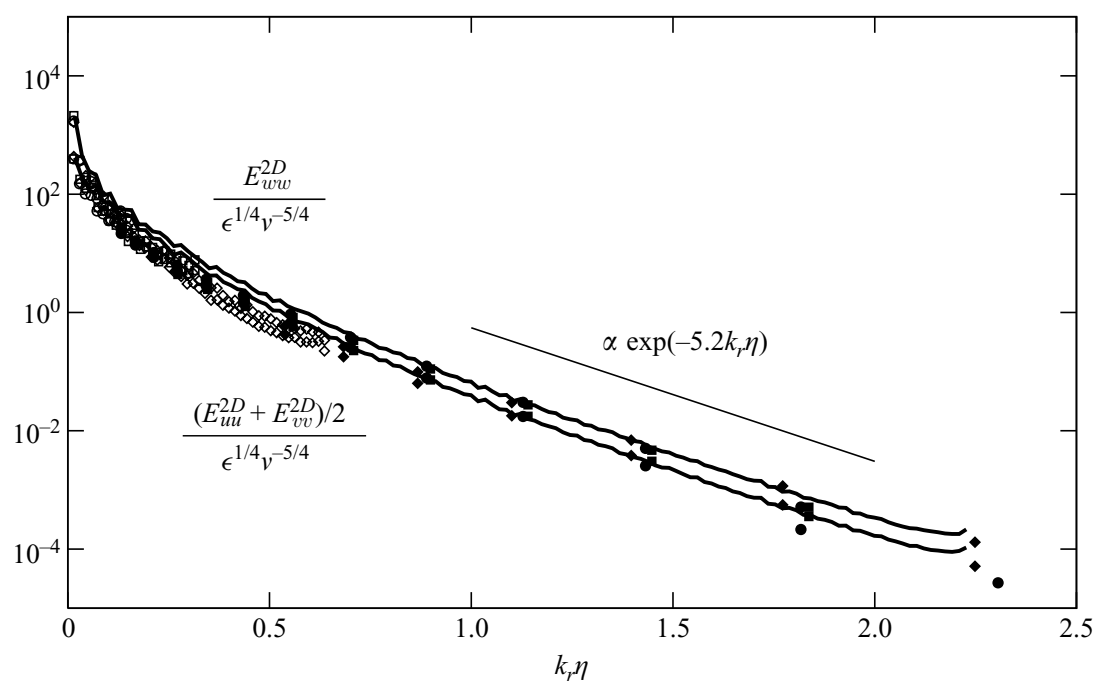

FIGURE 12. LES and DNS comparisons, with $\lambda_{v}=1.61 \widetilde{\eta}$, of mid-plane compensated velocity spectra in log-linear coordinates: open symbols, resolved; solid symbols, subgrid; see table 2 for key.

That the anisotropy increases with wavenumber is perhaps counterintuitive, and perhaps contradictory to the notion of small-scale isotropy. This was first pointed out by Livescu \& Ristorcelli (2008) and further discussed by Livescu et al. (2009). Cabot $\&$ Cook (2006) measured small-scale anisotropy by comparing the relative magnitudes of directional Kolmogorov microscales, defined by $\eta_{i}=\left(v^{3} / \epsilon_{i}\right)^{1 / 4}, \epsilon_{i}=15 v\left\langle\left(\partial u_{i} / \partial x_{i}\right)^{2}\right\rangle$ (no summation), but found that, with this measure, the small scales were isotropic. A question then arises as to why the velocity-anisotropy parameter increases in the viscosity-dominated range $10^{-1}<k_{r} \eta<2$. Since all components of velocity fluctuations are decreasing in this range (figure 11a), the anisotropy can only increase if the horizontal velocity fluctuations decrease faster than the vertical velocity fluctuations. According to Livescu et al. (2009), this occurs because buoyancy production, which is anisotropic, exceeds both the nonlinear transfer and the approximately isotropic viscous dissipation.

The agreement between DNS and the present LES suggests that a special SGS model for active scalars is not needed for unstably stratified flows; a passive scalar SGS model is sufficient. This can be explained as follows: modelling each LES cell as a unit of stationary-homogeneous unstably stratified turbulence, we find that the subgrid buoyancy flux in each cell is equal to the its subgrid kinetic energy dissipation, but, since this dissipation is already accounted for in a passive scalar SGS model (by matching structure functions at the cutoff scale), we have also indirectly accounted for the subgrid buoyancy flux. In broad terms, it appears that statistically stationary unstable stratification does not disrupt the classical picture of turbulent mixing embodied in the Richardson cascade.

The DNS case E in table 1 requires 400 hours of wall clock time on 64 processors to collect statistics over 5 eddy-turnover times. In contrast, the lowest-resolution LES (case E1 in table 2) only requires 1 hour of wall clock time on four of the same processors to collect statistics over 23 eddy-turnover times. In terms of processorhours per eddy-turnover time, this represents savings by a factor in excess of $10^{4}$. 

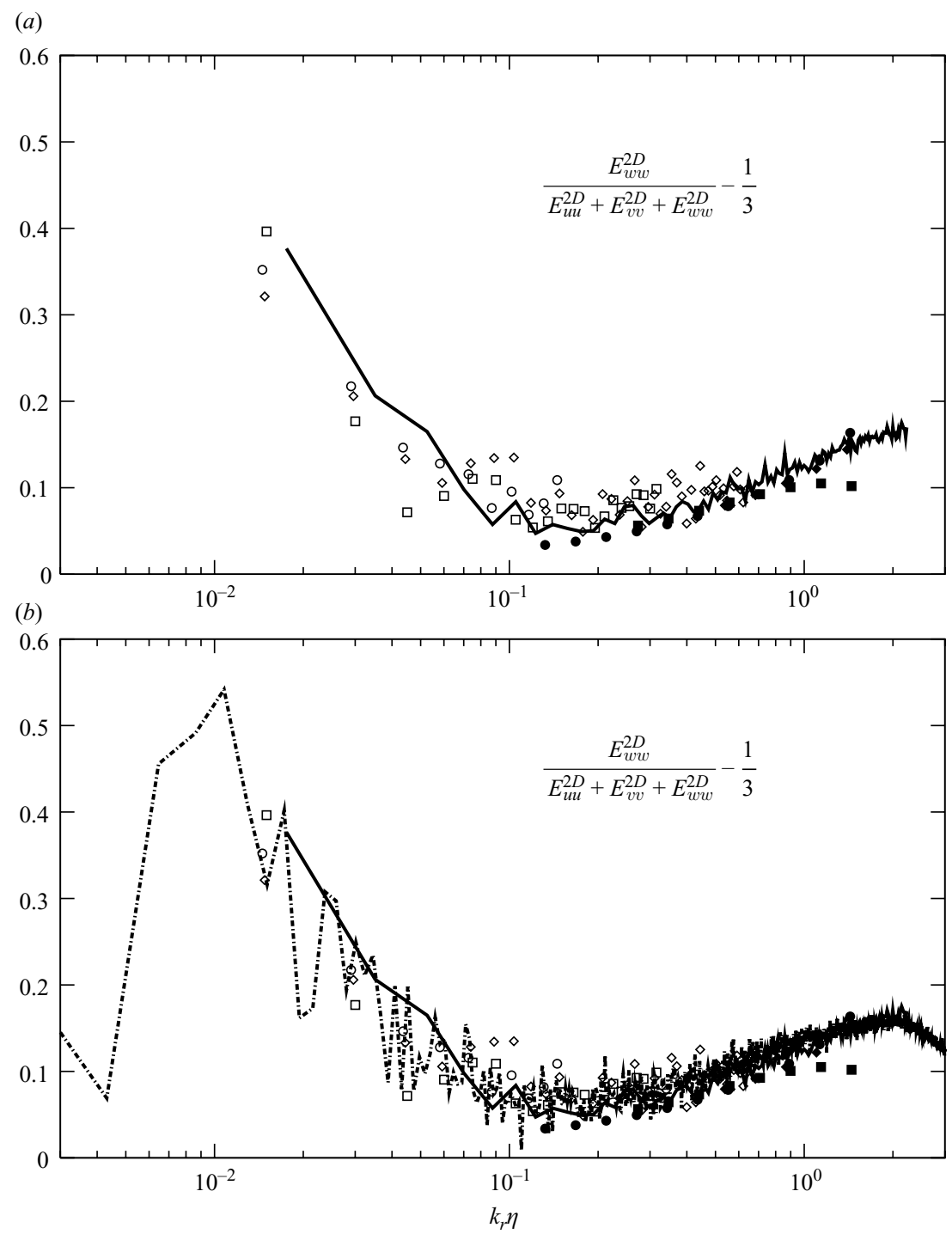

FIGURE 13. LES and DNS comparisons, with $\lambda_{v}=1.61 \widetilde{\eta}$, of the mid-plane velocity-anisotropy parameter: open symbols, resolved; solid symbols, subgrid; see table 2 for key; --.--.--, $3072^{3}$ DNS of Rayleigh-Taylor instability (Cabot \& Cook 2006).

\section{Conclusions}

To better understand the nature of buoyancy-driven turbulence, we have proposed a novel method to simulate statistically stationary buoyancy-driven turbulence. The main idea is to adapt the fringe-region technique to supply the flow with unmixed fluids within a triply periodic domain in the presence of gravity. The flow comprises an unforced mixing zone sandwiched between two thin horizontal fringe layers that inject unmixed fluids in an unstable configuration (heavy on top of light). At each point in the fringe, heavy fluid is introduced at the same mass rate as light fluid is removed so that no net mass, momentum or kinetic energy is introduced; that is the flow is purely driven by buoyancy, which, in turn, is generated by density fluctuations 
created in the fringes. The fringes could also be interpreted as 'unmixing' zones. This setup results in a statistically stationary flow, characterized by a linear mean mole fraction profile.

We also develop a method to integrate the variable-density incompressible flow equations in a way that satisfies discrete mass conservation (expressed as a constraint on the velocity divergence) regardless of iteration errors. This involves replacing the pressure with an alternative Lagrange multiplier, obtained from the Helmholtz-Hodge decomposition of $(1 / \rho)\left(\partial p / \partial x_{i}\right)$. When the governing equations are discretized in this form, iteration errors are isolated to the time integration of baroclinic vorticity.

The DNS has been performed at various $R e, A$ and aspect ratios $L_{z} / L_{x}$. For the $L_{z} / L_{x}$ investigated, namely $1 / 2,1$ and 2 , we find that the large eddies tend to fill the horizontal extent of the simulation domain: $l_{\rho} \approx 0.5 L_{x}$, where $l_{\rho}$ is the horizontal integral wavelength of the box based on density fluctuations. Consequently, this DNS should be interpreted as a model to study only the small scales of buoyancy-driven turbulence in the same way that we view DNS of homogeneous-isotropic turbulence.

When the computational domain is short relative to its width (case B), we report non-trivial correlations between the forced flow in the fringes and the flow at the mid-plane location, reducing the usefulness of physical generalizations that can be drawn from that particular case. Except for case B, the ratio of Taylor-microscale Reynolds numbers is found to be $R e_{\lambda_{z}} / R e_{\lambda_{x y}} \approx 2-2.5$, indicating sustained anisotropy. This is close to the values reported in the Rayleigh-Taylor instability simulations of Cook \& Dimotakis (2001) in which $R e_{\lambda_{z}} / R e_{\lambda_{x y}} \approx 2.5-4$.

All the present DNS mid-plane planar spectra, namely $E_{w w}^{2 D},\left(E_{u u}^{2 D}+E_{v v}^{2 D}\right) / 2, E_{\rho \rho}^{2 D}$ and $-E_{\rho w}^{2 D}$, collapse when scaled with the KOC scales: $\nu, \epsilon$ and $\epsilon_{\rho}$. In particular, they collapse regardless of the density ratio and the aspect ratio. For the Reynolds numbers considered, the spectra appear to exhibit about one decade of power-law range, where $E_{w w}^{2 D} \sim k_{r}^{-5 / 3},\left(E_{u u}^{2 D}+E_{v v}^{2 D}\right) / 2 \sim k_{r}^{-5 / 3}, E_{\rho \rho}^{2 D} \sim k_{r}^{-5 / 3}$ and $-E_{\rho w}^{2 D} \sim k_{r}^{-7 / 3}$. When compared with the corresponding spectra from the $3072^{3}$ DNS of Rayleigh-Taylor instability (Cabot \& Cook 2006), $E_{\rho \rho}^{2 D}$ and $-E_{\rho w}^{2 D}$ collapse, but not in the velocity spectra, where the present spectra show slightly more energy in the dissipation range $\left(k_{r} \eta>10^{-1}\right)$. This could perhaps be attributed to the difference between a statistically evolving flow, in which production exceeds dissipation (Rayleigh-Taylor), and a statistically stationary flow, in which production equals dissipation (present simulations).

Except for case B, the heavy-fluid mole fraction $\left(X_{2}\right)$ p.d.f.s plotted at various vertical locations within the mixing region (outside the fringe) exhibit a unimodal Gaussian-like distribution with peaks approaching $X_{2}=0$ near the lower fringe, and gradually shifting towards peaks approaching $X_{2}=1$ near the upper fringe, with a slight skew towards $X_{2}=0$ at the mid-plane location.

Using the DNS as an a posteriori test bed for the vortex-based SGS passive scalar flux model of Pullin (2000), we show that SGS model can be utilized to accurately predict buoyancy-driven turbulent mixing flows. It is remarkable that the numerical value of the $O(1)$ mixing-time constant, $\gamma_{Y_{1}}$, previously estimated by elementary means, agrees with the present calculations.

At a small fraction (down to $10^{-4}$ ) of the DNS computational effort, we have performed LES of the DNS case E in table 1. The LES spectra, including subgrid extensions, capture many essential features of the DNS spectra. For example, the LES velocity spectra exhibit large-scale anisotropy in the resolved component and also small-scale anisotropy in the subgrid extension, including the $\exp (-\beta k \eta)$ shape of the viscous roll-off. We also show that the SGS model of Pullin (2000) contains an intrinsic 
$-7 / 3$ power-law inertial-range scaling for the SGS $\rho-w$ cospectrum, consistent with the kind of scaling arguments articulated by Lumley (1967). We then use this to obtain the subgrid extension of $-E_{\rho w}^{2 D}$, which shows agreement with DNS. Furthermore, the LES velocity-anisotropy parameter, $E_{w w}^{2 D} / E_{u_{i} u_{i}}^{2 D}-1 / 3$, shows a minimum at the beginning of the dissipation range, $k_{r} \eta=10^{-1}$, in agreement with the present DNS, and also the $3072^{3}$ Rayleigh-Taylor DNS of Cabot \& Cook (2006). The LES-DNS agreement demonstrates that the SGS model of Pullin (2000), originally designed for passive scalars, can be used without modifications for statistically stationary unstably stratified turbulence. This is because unstably stratified flows are rate limited by turbulent mixing, a key argument used in the development of the passive scalar SGS model.

The authors wish to thank Drs. W. H. Cabot and A. W. Cook for kindly providing their DNS spectra. This work is partially supported by the NSF under grant CBET 0651754 .

\section{Appendix. Numerical method}

The governing equations, in the form (2.7), (2.8) and (3.1), are discretized using the low-storage semi-implicit Runge-Kutta method of Spalart et al. (1991). Briefly, the method consists of three sequential substeps of the following form:

$$
\begin{aligned}
\frac{s^{(n+1)}-s^{(n)}}{\Delta_{t}}= & \gamma H_{s}^{(n)}+\zeta H_{s}^{(n-1)}+\alpha \mathscr{D} \frac{\partial^{2}}{\partial x_{j}^{2}} s^{(n)}+\beta \mathscr{D} \frac{\partial^{2}}{\partial x_{j}^{2}} s^{(n+1)}, \\
\frac{u_{i}^{(n+1)}-u_{i}^{(n)}}{\Delta_{t}}= & \gamma H_{i}^{(n)}+\zeta H_{i}^{(n-1)}+\alpha \frac{\mu}{\rho_{0}} \frac{\partial^{2}}{\partial x_{j}^{2}} u_{i}^{(n)}+\beta \frac{\mu}{\rho_{0}} \frac{\partial^{2}}{\partial x_{j}^{2}} u_{i}^{(n+1)} \\
& -\frac{(\alpha+\beta)}{\rho^{(*)}}\left(\frac{\partial p}{\partial x_{i}}+\Gamma_{i}\right), \\
\frac{\partial}{\partial x_{j}} u_{j}^{(n+1)}= & -\mathscr{D} \frac{\partial^{2}}{\partial x_{j}} s^{(n+1)}-\omega_{s}^{(n+1)},
\end{aligned}
$$

where

$$
\begin{aligned}
& H_{s}=-u_{j} \frac{\partial s}{\partial x_{j}}+\omega_{s}, \\
& H_{i}=-u_{j} \frac{\partial u_{i}}{\partial x_{j}}+\frac{\mu}{\rho_{0}}\left[\left(\frac{\rho_{0}}{\rho}-1\right) \frac{\partial^{2} u_{i}}{\partial x_{j}^{2}}+\frac{\rho_{0}}{\rho} \frac{1}{3} \frac{\partial}{\partial x_{i}} \frac{\partial u_{j}}{\partial x_{j}}\right]-g \delta_{i 3},
\end{aligned}
$$

$\rho \equiv \rho_{0} \exp (s),(\alpha+\beta) / \rho^{(*)} \equiv \alpha / \rho^{(n)}+\beta / \rho^{(n+1)}$, and $\omega_{s}$ is given by $(2.14 b)$. The values for $\alpha, \beta, \gamma$ and $\zeta$, different for each substep, are given by Spalart et al. (1991). For stability, we have chosen to split the viscous operator into the linear component, which we treat implicitly, and the nonlinear component, which we treat explicitly. Discretizing $s, \in(\infty, \infty)$, rather than $\rho$, ensures that $\rho>0$, but then, $[\rho]_{x}=\rho_{0}$ can no longer be maintained discretely; presently, we control this numerical drift with a small proportional control added to $\Lambda_{1}$ and $\Lambda_{2}$. The CFL is dynamically adjusted so that

$$
\Delta_{t}\left\{|u| / \Delta_{x},|v| / \Delta_{y},|w| / \Delta_{z}\right\}=0.7
$$

Presently, $\Delta_{x}=\Delta_{y}=\Delta_{z}$ everywhere. 
The spatial discretization of (A 1) employs the Fourier pseudospectral method (see Canuto et al. 1987), where the products and nonlinear terms in $H_{i}$ and $H_{s}$ are computed in physical space, then transformed to spectral space for the $2 / 3$-rule dealiasing. The 2/3-rule eliminates all aliasing errors arising from double products, but some higher-order aliasing from quotients and exponentials remains.

The steps for solving the system (A 1) are as follows. First, march (A 1a) one substep, then solve (A $1 b)$, choosing the Lagrange multipliers, $\left(\phi, \psi_{i}, f_{i}\right)$, where

$$
\frac{1}{\rho^{(*)}}\left(\frac{\partial p}{\partial x_{i}}+\Gamma_{i}\right) \equiv \frac{\partial \phi}{\partial x_{i}}+\epsilon_{i j k} \frac{\partial \psi_{k}}{\partial x_{j}}+f_{i}
$$

so that (A 1c) is discretely satisfied and that $\left\langle u_{i}^{n+1}\right\rangle_{m}=0$. The latter step of determining the Lagrange multipliers is described using continuous operators in $\S 3.1$.

\section{REFERENCES}

Batchelor, G. K., Canuto, V. M. \& Chasnov, J. R. 1992 Homogeneous buoyancy-generated turbulence. J. Fluid Mech. 235, 349-378.

Bertolotti, F. P., Herbert, T. \& Spalart, P. R. 1992 Linear and nonlinear stability of the Blasius boundary layer. J. Fluid Mech. 242, 441-474.

Савот, W. H. \& CoOK, A. W. 2006 Reynolds number effects on Rayleigh-Taylor instability with possible implications for type-Ia supernovae. Nature Phys. 2, 562-568.

Canuto, C., Hussaini, M. Y., Quarteroni, A. \& Zang, T. A. 1987 Spectral Methods in Fluid Dynamics. Springer.

Chang, W., Giraldo, F. \& Perot, B. 2002 Analysis of an exact fractional step method. J. Comput. Phys. 180, 183-199.

Cook, A. W., CABOt, W. \& Miller, P. L. 2004 The mixing transition in Rayleigh-Taylor instability. J. Fluid Mech. 511, 333-362.

Cook, A. W. \& Dimotakis, P. E. 2001 Transition stages of Rayleigh-Taylor instability between miscible fluids. J. Fluid Mech. 443, 69-99.

Dimotakis, P. E. 2005 Turbulent mixing. Annu. Rev. Fluid Mech. 37, 329-356.

Hill, D. J., Pantano, C. \& Pullin, D. I. 2006 Large-eddy simulation and multiscale modelling of a Richtmyer-Meshkov instability with reshock. J. Fluid Mech. 557, 29-61.

Krawczynski, J. F., Renou, B., Danaila, L. \& Demoulin, F. X. 2006 Small-scale measurements in a partially stirred reactor. Exp. Fluids 40, 667-682.

LivesCu, D. \& Ristorcelli, J. R. 2007 Buoyancy-driven variable-density turbulence. J. Fluid Mech. 591, 43-71.

Livescu, D. \& Ristorcelli, J. R. 2008 Variable-density mixing in buoyancy-driven turbulence. J. Fluid Mech. 605, 145-180.

Livescu, D., Ristorcelli, J. R., Gore, R. A., Dean, S. H., Cabot, W. H. \& Cook, A. W. 2009 High-Reynolds number Rayleigh-Taylor turbulence. J. Turbul. 10, N13.

Lumley, J. L. 1967 Similarity and the turbulent energy spectrum. Phys. Fluids 10, 855-858.

Lundgren, T. S. 1982 Strained spiral vortex model for turbulent fine structure. Phys. Fluids 25, 2193-2203.

Martínez, D. O., Chen, S., Doolen, G. D., Kraichnan, R. H., Wang, L.-P. \& Zhou, Y. 1997 Energy spectrum in the dissipation range of fluid turbulence. J. Plasma Phys. 57, 195-201.

Miller, P. L. 1991 Mixing in high Schmidt number turbulent jets. PhD thesis, California Institute of Technology, Pasadena.

Misra, A. \& Pullin, D. I. 1997 A vortex-based subgrid stress model for large-eddy simulation. Phys. Fluids 9, 2443-2454.

Mueschke, N. J. \& Schilling, O. 2009 Investigation of Rayleigh-Taylor turbulence and mixing using direct numerical simulation with experimentally measured initial conditions. I. Comparison to experimental data. Phys. Fluids 21, 014106. 
Nordström, J., Nordin, N. \& Henningson, D. 1999 The fringe region technique and the Fourier method used in the direct numerical simulation of spatially evolving viscous flows. SIAM J. Sci. Comput. 20, 1365-1393.

Overholt, M. R. \& Pope, S. B. 1996 Direct numerical simulation of a passive scalar with imposed mean gradient in isotropic turbulence. Phys. Fluids 8, 3128-3148.

Pullin, D. I. 2000 A vortex-based model for the subgrid flux of a passive scalar. Phys. Fluids 12, 2311-2319.

Pullin, D. I. \& Lundgren, T. S. 2001 Axial motion and scalar transport in stretched spiral vortices. Phys. Fluids 13, 2553-2563.

Pullin, D. I. \& Saffman, P. G. 1993 On the Lundgren-Townsend model of turbulent fine scales. Phys. Fluids A 5, 126-145.

Pullin, D. I. \& Saffman, P. G. 1994 Reynolds stresses and one-dimensional spectra for a vortex model of homogeneous anisotropic turbulence. Phys. Fluids 6, 1787-1796.

Saddoughi, S. G. \& Veeravalli, S. V. 1994 Local isotropy in turbulent boundary layers at high Reynolds number. J. Fluid Mech. 268, 333-372.

Sandoval, D. L. 1995 The dynamics of variable-density turbulence. PhD thesis, University of Washington, Seattle.

Schumacher, J. 2007 Sub-Kolmogorov-scale fluctuations in fluid turbulence. Europhys. Lett. 80, 54001.

Spalart, P. R., Moser, R. D. \& Rogers, M. M. 1991 Spectral methods for the Navier-Stokes equations with one infinite and two periodic directions. J. Comput. Phys. 96, 297-324.

Tong, Y., Lombeyda, S., Hirani, A. N. \& Desbrun, M. 2003 Discrete multiscale vector field decomposition. ACM Trans. Graphics 22, 445-452.

Voelkl, T., Pullin, D. I. \& Chan, D. C. 2000 A physical-space version of the stretched-vortex subgrid-stress model for large-eddy simulation. Phys. Fluids 12, 1810-1825.

Warhaft, Z. 2000 Passive scalars in turbulent flows. Annu. Rev. Fluid Mech. 32, 203-240.

Yeung, P. K., Donzis, D. A. \& Sreenivasan, K. R. 2005 High-Reynolds-number simulation of turbulent mixing. Phys. Fluids 17, 081703. 